\title{
Linear stability of fractional reaction - diffusion systems
}

\author{
Y. $\mathrm{Nec}^{1}$ and A. A. Nepomnyashchy \\ Department of Mathematics, Technion, Israel Institute of Technology, Haifa, 32000, Israel
}

\begin{abstract}
Theoretical framework for linear stability of an anomalous sub-diffusive activatorinhibitor system is set. Generalized Turing instability conditions are found to depend on anomaly exponents of various species. In addition to monotonous instability, known from normal diffusion, in an anomalous system oscillatory modes emerge. For equal anomaly exponents for both species the type of unstable modes is determined by the ratio of the reactants' diffusion coefficients. When the ratio exceeds its normal critical value, the monotonous modes become stable, whereas oscillatory instability persists until the anomalous critical value is also exceeded. An exact formula for the anomalous critical value is obtained. It is shown that in the short wave limit the growth rate is a power law of the wave number. When the anomaly exponents differ, disturbance modes are governed by power laws of the distinct exponents. If the difference between the diffusion anomaly exponents is small, the splitting of the power law exponents is absent at the leading order and emerges only as a next-order effect. In the short wave limit the onset of instability is governed by the anomaly exponents, whereas the ratio of diffusion coefficients influences the complex growth rates. When the exponent of the inhibitor is greater than that of the activator, the system is always unstable due to the inhibitor enhanced diffusion relatively to the activator. If the exponent of the activator is greater, the system is always stable. Existence of oscillatory unstable modes is also possible for waves of moderate length.
\end{abstract}

Key words: reaction-diffusion, anomaly exponent, monotonous instablity, oscillatory instability, critical diffusion coefficients' ratio.

AMS subject classification: 34D05, 35K57.

\footnotetext{
${ }^{1}$ Corresponding author. Email: flyby@techunix.technion.ac.il
} 


\section{Introduction}

Diffusive processes modelled by differential equations of fractional order have drawn intense interest in the last decade. Fractional derivatives granted the possibility to extend the innate scaling of Fickian diffusion $\left\langle r^{2}(t)\right\rangle \sim t$ to a more general property $\left\langle r^{2}(t)\right\rangle \sim t^{\gamma}$, thereby introducing the exponent $0<\gamma<1$ for anomalous sub-diffusion and $\gamma>1$ for superdiffusion ( see review [1] ). Sub-diffusion characterises systems with spatial or temporal constraints, significantly slowing down the transport process, like in porous media [2], gels [3], polymer solutions [4] and living cells [5, 6]. Super-diffusion arises when the system nature enables enhanced transport, like in turbulent flows [7, 8] or catalytic surface reactions $[9,10]$.

Occurrence of spatial ( Turing ) patterns in normal diffusive systems has long been described and understood, however a pertinent quest concerns the appearance of patterns with spatial periodicity in anomalous systems. Thus a substantially general stability theory is of a great interest. In this context several formal models have been suggested as a generalization of the normal reaction-diffusion equations. If the progress of a diffusion limited reaction is impeded by the same physical factors that hinder the diffusion, the reaction term has to be modified by application of a fractional derivative [11]. Then stability criteria are not affected by anomaly $[12,13]$. However, when the reaction is activation limited or mediated by additional normally diffusing reagents, diffusion anomaly will not interfere with its rate, thus leaving the reaction term unchanged. A generalized model of that type was derived in [15] via a continuous time random walk with memory and sources:

$$
\frac{\partial \mathbf{n}}{\partial t}=C \mathcal{D}^{1-\gamma} \nabla^{2} \mathbf{n}+\mathbf{f}(\mathbf{n}), \quad \mathcal{D}^{1-\gamma}=\mathfrak{D}^{1-\gamma}+\mathcal{L}^{-1}\left(\mathfrak{D}^{-\gamma}[\cdot]_{t=0}\right),
$$

wherein $\mathbf{n}(\mathbf{r}, t)$ is the vector of density numbers of the reacting components, depending on position $\mathbf{r}$ and time $t$, and $\mathbf{f}(\mathbf{n}(\mathbf{r}, t))$ determines the reaction kinetics. $C$ is a diagonal matrix of diffusion coefficients, assumed constant. The anomaly exponent $0<\gamma<1$ is generally different for each species. The operator

$$
\mathfrak{D}^{-\gamma} y(t)=\frac{d^{-\gamma} y(t)}{d t^{-\gamma}}=\frac{1}{\Gamma(\gamma)} \int_{0}^{t} \frac{y(\tau)}{(t-\tau)^{1-\gamma}} d \tau
$$

denotes the Riemann-Liouville fractional integral and

$$
\mathfrak{D}^{1-\gamma} y(t)=\frac{d^{1-\gamma} y(t)}{d t^{1-\gamma}}=\frac{d}{d t} \frac{d^{-\gamma} y(t)}{d t^{-\gamma}} .
$$

$\mathcal{L}^{-1}$ denotes the inverse Laplace transform. The term $\mathcal{L}^{-1}\left(\mathfrak{D}^{-\gamma}[\cdot]_{t=0}\right)$ is essential due to the singular nature of the fractional derivative and prevents introduction of unphysical diverging terms upon Laplace transform of (1.1). At the limit $\gamma \rightarrow 1$ the regularization term vanishes, as inverse Laplace transform of an entire function would yield a zero residue, and (1.1) reduces to the normal reaction-diffusion equation. It should be noted that there exists an alternative definition of the fractional derivative $[1,14]$, where the regularization is intrinsic:

$$
\mathfrak{D}_{*}^{1-\gamma} y(t)=\frac{1}{\Gamma(\gamma)} \int_{0}^{t} \frac{d y(\tau) / d \tau}{(t-\tau)^{1-\gamma}} d \tau .
$$


Note that the use of $\mathfrak{D}_{*}$ is restricted to differentiable functions only. Operators $\mathfrak{D}_{*}$ and $\mathfrak{D}$ are related by

$$
\mathfrak{D}_{*}^{1-\gamma} y(t)=\mathfrak{D}^{1-\gamma}\left(y(t)-y\left(0^{+}\right)\right)
$$

wherefrom follows that Laplace transform of $\mathfrak{D}_{*}$ will contain initial condition dependent terms. Obviously, at the asymptotic limit $t \rightarrow \infty$ exponentially growing modes will render these terms negligible. Thus unstable modes acquired via dispersion relation with either of the operators are equivalent. Operator (1.3) is therefore preferred in this context for the mathematical convenience granted by no dependence on initial conditions and the wider class of candidate solution functions it allows.

The model was partly investigated with specific values of anomaly exponents and exhibited intriguing properties $[15,16]$, in particular appearance of oscillatory unstable modes, unexistent in normal and fractional systems with the fractional derivative acting on both reaction and diffusion terms $[12,13]$. The current investigation is aimed to complete the linear stability analysis for a two species rotationally invariant sub-diffusive system with general anomaly exponents and normal reaction. Conditions for Turing instability are generalized in section 3 and unstable modes of monotonous and oscillatory nature are located in sections 4 and 5 for equal and generally distinct exponents respectively. Asymptotic behavior of the growth rate for long and short waves is analyzed, and an exact formula for the critical diffusion coefficients' ratio is obtained ( where exists). Concluding remarks appear in section 6.

\section{Formulation}

A two-species fractional activator-inhibitor system is described, as a special case of (1.1) by the following system of equations

$$
\begin{gathered}
\frac{\partial \mathbf{n}}{\partial t}=C \mathcal{D}^{1-\gamma} \nabla^{2} \mathbf{n}+\mathbf{f}(\mathbf{n}) \text { in } \Omega \\
\mathbf{n}=\left(\begin{array}{l}
n_{1} \\
n_{2}
\end{array}\right), \quad C=\left(\begin{array}{ll}
1 & 0 \\
0 & d
\end{array}\right), \quad \mathcal{D}^{1-\gamma} \mathbf{n}=\left(\begin{array}{c}
\mathcal{D}^{1-\gamma_{1}} n_{1} \\
\mathcal{D}^{1-\gamma_{2}} n_{2}
\end{array}\right), \quad \mathbf{f}=\left(\begin{array}{c}
f_{1} \\
f_{2}
\end{array}\right), \\
n_{j}=n_{j}(\mathbf{r}, t), \quad f_{j}=f_{j}\left(n_{1}, n_{2}\right), \quad j \in\{1,2\},
\end{gathered}
$$

where $n_{1}$ and $n_{2}$ are respectively activator and inhibitor density numbers, and $d$ is the diffusion coefficients' ratio. For simplicity, the domain $\Omega$ is the whole space or has a rectangular shape,

$$
\Omega \subset \mathbb{R}^{p}, \quad p \in\{1,2,3\} .
$$

Initial condition $\mathbf{n}(\mathbf{r}, 0)=\left.\mathbf{n}\right|_{t=0}$ is prescribed. To complete the formulation of (2.1), the boundary conditions are taken either periodic or zero flux across the domain boundary $\nabla \mathbf{n} \cdot \nu=0$ on $\partial \Omega(\nu$ is the outward normal $)$.

Temporal evolution of a small perturbation $\Delta \mathbf{n}$ about a uniform steady state $\mathbf{n}_{0}$ is governed by the linearized system 


$$
\frac{\partial}{\partial t} \Delta \mathbf{n}=C \mathcal{D}^{1-\gamma} \nabla^{2}(\Delta \mathbf{n})+\nabla \mathbf{f} \Delta \mathbf{n}
$$

wherein

$$
\nabla \mathbf{f}_{j k}=\left(\frac{\partial f_{j}}{\partial n_{k}}\right)_{\mathbf{n}_{0}}
$$

is the kinetic sensitivity matrix. Conventionally, for $\mathbf{n}_{0}$ to be stable to spatially uniform disturbances the entries of $\nabla \mathbf{f}$ obey [17]

$$
\begin{gathered}
\operatorname{tr} \nabla \mathbf{f}=\nabla \mathbf{f}_{11}+\nabla \mathbf{f}_{22}<0, \quad \operatorname{tr}_{w} \nabla \mathbf{f}=d \nabla \mathbf{f}_{11}+\nabla \mathbf{f}_{22}>0, \quad \operatorname{det} \nabla \mathbf{f}>0, \\
\nabla \mathbf{f}_{11}>0, \quad \nabla \mathbf{f}_{22}<0, \quad d>1 .
\end{gathered}
$$

Upon subsequent application of temporal Laplace transform, denoted by tilde, and spatial Fourier transform, denoted by hat, (2.3) becomes

$$
\widehat{\widehat{\Delta \mathbf{n}}}=\left.S_{\mathcal{L}}^{-1} \widehat{\Delta \mathbf{n}}\right|_{t=0}
$$

where

$$
S_{\mathcal{L}}=\left(\begin{array}{cc}
s-\nabla \mathbf{f}_{11}+q^{2} s^{1-\gamma_{1}} & -\nabla \mathbf{f}_{12} \\
-\nabla \mathbf{f}_{21} & s-\nabla \mathbf{f}_{22}+d q^{2} s^{1-\gamma_{2}}
\end{array}\right)
$$

with $s$ and $\mathbf{q} \in \mathbb{R}^{p}$ being Laplace and Fourier transform variables respectively, $q=|\mathbf{q}|$. If the domain $\Omega$ is infinite, appropriate decay of $\mathbf{n}(\mathbf{r}, t)$ is assumed for $|\mathbf{r}| \rightarrow \infty$, and the spectrum $\mathbf{q}$ is continuous. Otherwise, both for zero-flux and periodic boundary conditions the spectrum is discrete.

Application of the inverse Laplace transform reveals that the temporal evolution is determined by the roots of $D\left(q, s ; \gamma_{1}, \gamma_{2}\right)$, the determinant of $S_{\mathcal{L}}$. Hence the dispersion relation is given by

$$
D\left(q, s ; \gamma_{1}, \gamma_{2}\right)=\operatorname{det} S_{\mathcal{L}}=0
$$

\section{Roots entailing instability}

Turing patterns are evoked by diffusion driven instability, whereas the steady state $\mathbf{n}_{0}$ is stable in the absence of diffusion. Equivalently, when $\mathbf{q}=0$, both for normal and anomalous diffusion stability of $\mathbf{n}_{0}$ ensues when ( e.g. $[15,17]$ )

$$
\Re s<0, \quad D\left(0, s ; \gamma_{1}, \gamma_{2}\right)=0 .
$$

For $q \neq 0$, equation (2.8) possesses two roots if $\gamma_{1}=\gamma_{2}=1$ and generally many roots otherwise. In the latter event existence of roots with positive real part does not necessarily lead to instability [16]. Therefore, further analysis of the disturbance temporal evolution 
is required to characterize the truly unstable roots. Let us first analyze the case of equal anomaly exponents for both reactants $\gamma_{1}=\gamma_{2}=\gamma$, where

$$
D(q, s ; \gamma)=s^{2}+(1+d) q^{2} s^{2-\gamma}-\operatorname{tr} \nabla \mathbf{f} s+d q^{4} s^{2(1-\gamma)}-q^{2} \operatorname{tr}_{w} \nabla \mathbf{f} s^{1-\gamma}+\operatorname{det} \nabla \mathbf{f} .
$$

To examine the roots lying on different branches of the Riemann surface, without loss of generality $\gamma$ is taken to be a simple reduced fraction $\gamma=m / r$, and a new variable is introduced

$$
\sigma=s^{1 / r}=|s|^{1 / r} e^{i \arg (s) / r} .
$$

Then temporal growth ensues if there exists a root located within a certain sector:

$$
\begin{aligned}
& \text { (i) } \Re \sigma^{r}>0 \Leftrightarrow \Re s>0 \\
& \text { (ii) }-\frac{\pi}{r}<\arg \sigma<\frac{\pi}{r} \Leftrightarrow \quad-\frac{\pi}{r}<\arg s^{1 / r}<\frac{\pi}{r} .
\end{aligned}
$$

Conditions (3.4i) and (3.4ii) can be reformulated as

$$
-\frac{\pi}{2 r}<\arg s^{1 / r}<\frac{\pi}{2 r}
$$

In the special case of $\gamma=\frac{1}{2}$ the corresponding condition has been derived in [16].

When the anomaly exponents differ, by (2.8)

$$
\begin{aligned}
D\left(q, s ; \gamma_{1}, \gamma_{2}\right)= & s^{2}+d q^{2} s^{2-\gamma_{2}}+q^{2} s^{2-\gamma_{1}}-\operatorname{tr} \nabla \mathbf{f} s+d q^{4} s^{2-\gamma_{1}-\gamma_{2}}- \\
& d \nabla \mathbf{f}_{11} q^{2} s^{1-\gamma_{2}}-\nabla \mathbf{f}_{22} q^{2} s^{1-\gamma_{1}}+\operatorname{det} \nabla \mathbf{f} .
\end{aligned}
$$

Again, taking $\gamma_{j}=m_{j} / r_{j}, j \in\{1,2\}$ to be reduced fractions,

$$
\sigma=s^{1 / \mathcal{R}}
$$

where $\mathcal{R}$ is the least common denominator of both exponents and $D\left(q, \sigma ; \gamma_{1}, \gamma_{2}\right)$ possesses $2 \mathcal{R}$ roots. Consequently, the instability condition becomes

$$
-\frac{\pi}{2 \mathcal{R}}<\arg s^{1 / \mathcal{R}}<\frac{\pi}{2 \mathcal{R}}
$$

generalizing (3.5). Detailed derivation appears in appendix A.

\section{Stability in the case of equal anomaly exponents}

In the following analysis it is convenient to keep fixed the parameters $\operatorname{tr} \nabla \mathbf{f}, \operatorname{tr}_{w} \nabla \mathbf{f}$ and det $\nabla \mathbf{f}$, differently from the usual approach where the entries of $\nabla \mathbf{f}$ are fixed and $\operatorname{tr}_{w} \nabla \mathbf{f}$ varies accordingly with $d$. 
The curve $s(q ; d, \gamma)$, solving $D(q, s ; \gamma)=0$, is investigated to explore the nature of unstable modes. By preliminary asymptotic analysis, for large values of $|s|$ ( and $|\sigma|$ ) the implicit solution of $D(q, s ; \gamma)=0$

$$
\begin{gathered}
q_{ \pm}^{2}=\left(2 d \sigma^{r-m}\right)^{-1}\left\{-\left[\sigma^{r}(1+d)-\operatorname{tr}_{w} \nabla \mathbf{f}\right] \pm \sqrt{\Delta_{\sigma}}\right\} \\
\Delta_{\sigma}=\left[\sigma^{r}(1+d)-\operatorname{tr}_{w} \nabla \mathbf{f}\right]^{2}-4 d\left(\sigma^{2 r}-\operatorname{tr} \nabla \mathbf{f} \sigma^{r}+\operatorname{det} \nabla \mathbf{f}\right)
\end{gathered}
$$

simplifies to two branches

$$
\sigma \sim q^{2 / m}\left(\begin{array}{l}
1 \\
d^{1 / m}
\end{array}\right) e^{i \pi(1+2 j) / m}, \quad j=0, \ldots, m-1 .
$$

None of the roots can satisfy (3.5). Therefore for large values of $|s|$, corresponding to short evolution time, no instability can be observed. To paraphrase, the set of unstable roots is always bounded from above.

For small values of $|s|$ ( and $|\sigma|)$

$$
q_{ \pm}^{2} \sim \frac{\operatorname{tr}_{w} \nabla \mathbf{f}}{2 d \sigma^{r-m}}\left(1 \pm \sqrt{1-4 d \frac{\operatorname{det} \nabla \mathbf{f}}{\operatorname{tr}_{w}^{2} \nabla \mathbf{f}}}\right) .
$$

Because by definition equation (4.3) is valid only when $q \in \mathbb{R}$, the diffusion coefficients' ratio is below its normal critical value,

$$
d<d_{M}=\operatorname{tr}_{w}^{2} \nabla \mathbf{f} /(4 \operatorname{det} \nabla \mathbf{f}) .
$$

Hence $q_{ \pm}^{2} \sigma^{r-m}$ is real and positive, yielding

$$
\arg \sigma=\frac{2 \pi j}{r-m}, \quad j=0, \ldots, r-m-1 .
$$

For $j=0 \quad \arg \sigma=0$ and $\Re \sigma^{r}>0$, so that for small values of $|s|$, corresponding to long evolution time, a monotonous unstable mode is revealed. For $j=1$ the root lies outside of the sector:

$$
\frac{2 \pi}{r-m}>\frac{\pi}{r}
$$

Since the last root $(j=r-m-1)$ is located symmetrically below the real axis, it also lies outside the sector. Note that instability ensued for $d<d_{M} \operatorname{since}_{\operatorname{tr}_{w}} \nabla \mathbf{f}$ was taken fixed ( conversely to the prevailing approach, when $d$ is taken fixed and $\operatorname{tr}_{w} \nabla \mathbf{f}$ varies accordingly ).

\subsection{Region $d<d_{M}$}

When the anomaly exponents equal, the real branches of $s(q ; d, \gamma)$ are traced defining a stretched abscissa coordinate $q_{s}^{2}=s^{1-\gamma} q^{2}$ and transformation of (3.2) into

$$
D\left(q_{s}, s ; \gamma\right)=s^{2}+s\left[q_{s}^{2}(1+d)-\operatorname{tr} \nabla \mathbf{f}\right]+d q_{s}^{4}-\operatorname{tr}_{w} \nabla \mathbf{f} q_{s}^{2}+\operatorname{det} \nabla \mathbf{f},
$$


whose positive root is given by

$$
\begin{gathered}
s\left(q_{s}\right)=\frac{1}{2}\left\{-\left[q_{s}^{2}(1+d)-\operatorname{tr} \nabla \mathbf{f}\right]+\sqrt{\Delta_{q_{s}}}\right\} \\
\Delta_{q_{s}}=\left[q_{s}^{2}(1+d)-\operatorname{tr} \nabla \mathbf{f}\right]^{2}-4\left(d q_{s}^{4}-\operatorname{tr}_{w} \nabla \mathbf{f} q_{s}^{2}+\operatorname{det} \nabla \mathbf{f}\right) .
\end{gathered}
$$

Note that for normal diffusion $D\left(q_{s}, s\right)=D(q, s)$ [17]. Analysis of the roots of

$$
\begin{gathered}
d q_{s}^{4}-\operatorname{tr}_{w} \nabla \mathbf{f} q_{s}^{2}+\operatorname{det} \nabla \mathbf{f}=0, \\
q_{s_{ \pm}}^{2}=\frac{1}{2 d}\left(\operatorname{tr}_{w} \nabla \mathbf{f} \pm \sqrt{\left.\operatorname{tr}_{w}^{2} \nabla \mathbf{f}-4 d \operatorname{det} \nabla \mathbf{f}\right)}\right)
\end{gathered}
$$

implies that $s\left(q_{s_{ \pm}}\right)=0, s\left(q_{s_{-}}<q_{s}<q_{s_{+}}\right)>0$ and $s<0$ otherwise. Since $s \rightarrow 0$ whilst $q_{s} \rightarrow q_{s_{ \pm}}$means $q \rightarrow \infty$, there are two real decaying branches. Linearizing (4.8) about $q_{s_{ \pm}}$ gives the asymptotic behavior of $s(q)$ :

$$
\begin{gathered}
q \sim s^{(\gamma-1) / 2}\left(q_{s_{ \pm}}+s\left(2 q_{s_{ \pm}}\left(\frac{d s}{d q_{s}^{2}}\right)_{q_{s_{ \pm}}}\right)^{-1}\right), \\
\left.\frac{d s}{d q_{s}^{2}}\right|_{q_{s_{ \pm}}}=\frac{1}{2}\left(-(1+d)+\frac{q_{s_{ \pm}}^{2}(1-d)^{2}-(1+d) \operatorname{tr} \nabla \mathbf{f}+2 \operatorname{tr}_{w} \nabla \mathbf{f}}{q_{s_{ \pm}}^{2}(1+d)-\operatorname{tr} \nabla \mathbf{f}}\right),
\end{gathered}
$$

where $q_{s_{ \pm}}$give respectively the lower and the upper branches.

Similarly, the behavior around the maximum is approximated by Taylor series up to the quadratic term:

$$
\begin{gathered}
q \sim s^{(\gamma-1) / 2}\left(q_{\max } \pm \sqrt{\tilde{s}}\right) \tilde{s}=\frac{s-s_{\max }}{b}, \quad b:=2 q_{\max }^{2}\left(\frac{d^{2} s}{d\left(q^{2}\right)^{2}}\right)_{q_{\max }^{2}} \\
q_{\max }=\frac{1}{d(1-d)^{2}}\left(d\left[(1+d) \operatorname{tr} \nabla \mathbf{f}-2 \operatorname{tr}_{w} \nabla \mathbf{f}\right]+(1+d) \sqrt{\Delta_{q_{\max }}}\right) \\
s_{\max }=\frac{1}{(1-d)^{2}}\left((1+d) \operatorname{tr}_{w} \nabla \mathbf{f}-2 d \operatorname{tr} \nabla \mathbf{f}-2 \sqrt{\Delta_{q_{\max }}}\right) \\
\Delta_{q_{\max }}=d\left(\left(\operatorname{tr}_{w} \nabla \mathbf{f}-\operatorname{tr} \nabla \mathbf{f}\right)\left(\operatorname{tr}_{w} \nabla \mathbf{f}-d \operatorname{tr} \nabla \mathbf{f}\right)+(1-d)^{2} \operatorname{det} \nabla \mathbf{f}\right)
\end{gathered}
$$

Formula (4.11b) ensues by differentiation of (4.8), (4.11c) - by solving $D\left(q_{s}, s ; \gamma\right)=0$ as a quadratic equation for $q_{s}^{2}$ and equating the discriminant to zero. Choice of the correct quadratic root is based on the relation $d<d_{M}$. Note that $d \neq 1$ [17].

The two real branches $s(q)$ merge at the point $\left(q_{m}, s_{m}\right)$, the minimum of the single valued curve $q(s)$. A fourth-degree polynomial is encountered upon seeking a formal minimum, whereas the minimum of (4.11a) is found explicitly via the quadratic equation

$$
\gamma^{2} \tilde{s}^{2}+\left(\frac{2 \gamma}{b}-(1-\gamma)^{2} q_{\max }^{2}\right) \tilde{s}+\frac{s_{\max }^{2}}{b^{2}}=0
$$


The smaller root is chosen to keep $s_{m}$ positive:

$$
s_{m}=b \tilde{s}+s_{\max }, \quad b<0, \quad \tilde{s}>0 .
$$

$q_{m}$ is then obtained from (4.11a). Figure 1 depicts a typical solution and corresponding approximated curves and merge point.

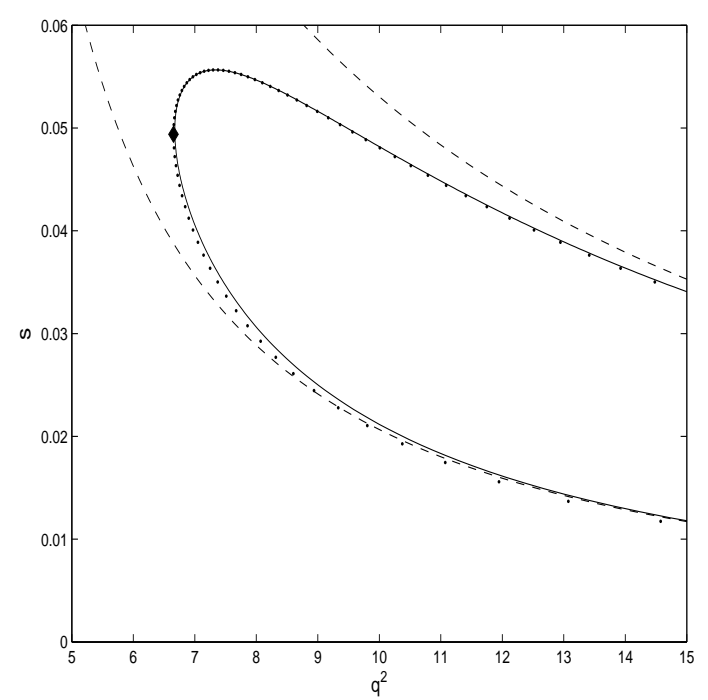

Figure 1: Sub-critical real branch ( $\operatorname{det} \nabla \mathbf{f}=0.3, \operatorname{tr} \nabla \mathbf{f}=-0.5, \operatorname{tr}_{w} \nabla \mathbf{f}=1.5, \gamma=\frac{1}{5}$, $d=\frac{3}{4} d_{M}$ ): numerical solution ( solid ), upper and lower asymptotics ( dashed ), second order Taylor approximation ( dotted ) and merge point $\left(q_{m}^{2}, s_{m}\right)$ ( diamond ).

For wave numbers $0<q<q_{m} \quad s \in \mathbb{C}$. Since $D(q, \sigma ; \gamma)$ is a polynomial with real coefficients, all complex roots are conjugate pairs. Hereinafter the root with positive imaginary part only is assessed. Since $\operatorname{tr} \nabla \mathbf{f}<0$,

$$
\left.s\right|_{q=0}=\frac{1}{2}\left(\operatorname{tr} \nabla \mathbf{f}+\sqrt{\Delta_{q=0}}\right), \quad \Delta_{q=0}:=\operatorname{tr}^{2} \nabla \mathbf{f}-4 \operatorname{det} \nabla \mathbf{f}
$$

has negative real part. If the discriminant $\Delta_{q=0}<0, \Re s$ ascends from $\frac{1}{2} \operatorname{tr} \nabla \mathbf{f}$ at $q=0$ to a positive value $s_{m}$ at $q_{m}$, and $\Im s$ changes from its maximal value $\frac{1}{2} \sqrt{-\Delta_{q=0}}$ at $q=0$ to zero at $q_{m}$. If however, $\Delta_{q=0}>0$, for $q \in\left(0, q_{m}\right)$ the roots are still complex conjugates, whereas at $q=0$ two real roots emerge. Then $\Im s$ attains its maximum within $\left(0, q_{m}\right)$ and vanishes at $q=0$.

Regardless of the behavior of $\Im s$ there exists a range $\left(q_{0}, q_{m}\right)$ where $\Re s>0$, and the corresponding modes are unstable. Detailed argument is brought in appendix B. A typical complex branch and a polar map of $s$ and $\sigma$ are shown in figures 2 and 3 respectively.

\subsection{Region $d>d_{M}$}

As the activator-inhibitor diffusion coefficients' ratio $d$ approaches the critical value of the normal diffusion problem $d_{M}$, the system becomes less unstable in the sense that the unstable 

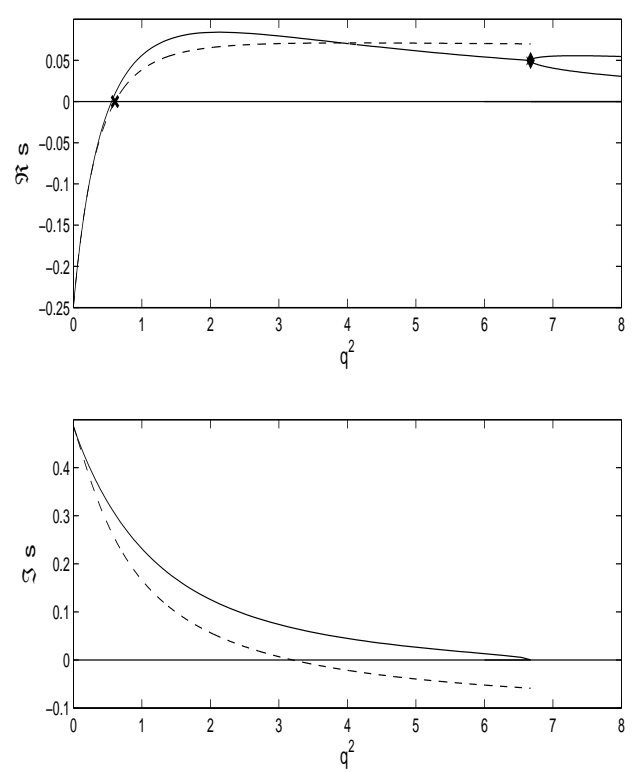

Figure 2: Sub-critical complex branch ( $\operatorname{det} \nabla \mathbf{f}=0.3, \operatorname{tr} \nabla \mathbf{f}=-0.5, \operatorname{tr}_{w} \nabla \mathbf{f}=1.5, \gamma=\frac{1}{5}$, $\left.d=\frac{3}{4} d_{M}\right)$ : numerical solution ( solid ), linear approximation ( dashed ), merge point $\left(q_{m}^{2}, s_{m}\right)$ ( diamond ) and minimal unstable wave $q_{0}^{2}$ ( x mark ).

modes grow slower. By (4.9) at $d=d_{M}$

$$
q_{s_{1}}=q_{s_{2}}=q_{s_{\max }}=\frac{1}{2 d} \operatorname{tr}_{w} \nabla \mathbf{f}
$$

whereas $s_{\max }=0$. Hence $q_{\max } \rightarrow \infty$, i.e. the double real branch merges into the real line, while shifting to infinity. The complex branch undergoes a similar flattening, yet retains all basic properties. Suppose

$$
\frac{d}{d_{M}}>1, \quad \frac{d}{d_{M}} \ngtr 1, \quad q^{2} \gg 1, \quad|s| \ll 1, \quad\left|q^{2} s^{1-\gamma}\right| \sim O(1) .
$$

This set of assumptions allows for a series expansion, capturing the behaviour at large wave numbers, when the real branch has already disappeared. Formally,

$$
s \sim \sum_{j=1}^{\infty} w_{j} q^{2 \mu j}, \quad w_{j} \sim O(1)
$$

where $\mu<0$ is a constant to be determined. Substitution into (3.2) and scrutiny of the powers of $q$ yields

$$
\mu=-\frac{1}{1-\gamma}
$$

Solving $O(1)$ equation

$$
d w_{1}^{2(1-\gamma)}-\operatorname{tr}_{w} \nabla \mathbf{f} w_{1}^{1-\gamma}+\operatorname{det} \nabla \mathbf{f}=0
$$



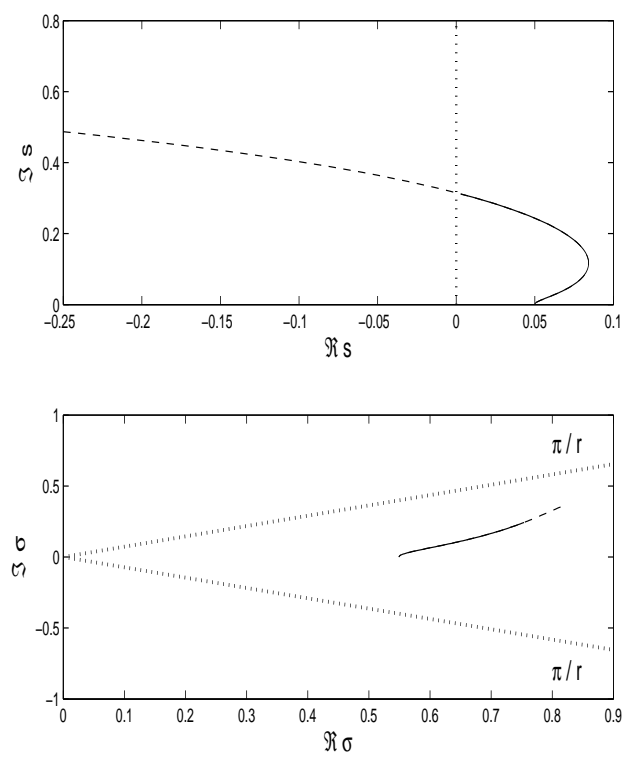

Figure 3: Sub-critical polar map, $0<q<q_{m}\left(\operatorname{det} \nabla \mathbf{f}=0.3, \operatorname{tr} \nabla \mathbf{f}=-0.5, \operatorname{tr}_{w} \nabla \mathbf{f}=1.5\right.$, $\left.\gamma=\frac{1}{5}, d=\frac{3}{4} d_{M}\right)$ : unstable waves $q_{0}<q<q_{m}$ ( solid ) and stable waves $0<q<q_{0}$ (dashed).

gives

$$
w_{1}^{1-\gamma}=\frac{\operatorname{tr}_{w} \nabla \mathbf{f}}{2 d}\left(1+i \sqrt{\frac{d}{d_{M}}-1}\right)
$$

and

$$
\arg s \sim \frac{1}{1-\gamma} \arctan \sqrt{\frac{d}{d_{M}}-1} \in\left(0, \frac{\pi}{2}\right) .
$$

Now allowing $d / d_{M} \gg 1$ permits to obtain the anomalous critical value $d_{M}$, where $\arg s \sim \frac{\pi}{2}$. To first order,

$$
d_{O} \sim d_{M} \sin ^{-2} \frac{\gamma \pi}{2}
$$

An explicit expression for the second order correction is computed from $O\left(q^{\mu}\right)$ equation ( after some algebra )

$$
\begin{gathered}
w_{2}=-\frac{1}{1-\gamma}\left(\frac{\operatorname{det} \nabla \mathbf{f}}{d}\right)^{1 /(1-\gamma)}\left(\frac{\frac{d}{d_{M}} \operatorname{tr}^{2} \nabla \mathbf{f}-2(1+d) \operatorname{tr} \nabla \mathbf{f}+(1+d)^{2}}{\frac{d}{d_{M}}-1}\right)^{1 / 2} \times \\
\exp \left[i\left(\frac{2}{1-\gamma} \arctan \sqrt{\frac{d}{d_{M}}-1}+\arctan \frac{\operatorname{tr} \nabla \mathbf{f}-(1+d)}{\operatorname{tr} \nabla \mathbf{f} \sqrt{\frac{d}{d_{M}}-1}}\right)\right] .
\end{gathered}
$$


Inspection of (4.23) immediately yields that (4.22) is exact to second order. By (4.17) all terms in the expansion

$$
\tan \arg s \sim \frac{\sum_{j=1}^{\infty} \Im w_{j} q^{2 \mu j}}{\sum_{j=1}^{\infty} \Re w_{j} q^{2 \mu j}} \sim \frac{\Im w_{1}}{\Re w_{1}}+q^{2 \mu} \frac{\Re w_{1} \Im w_{2}-\Im w_{1} \Re w_{2}}{\left(\Re w_{1}\right)^{2}}+O\left(q^{4 \mu}\right),
$$

diverge for $\Re w_{1}=0$, and hence the anomalous critical diffusion coefficients' ratio is given exactly by

$$
d_{O}=d_{M} \sin ^{-2} \frac{\gamma \pi}{2}
$$

Identical formula is obtained when seeking a value $d$, where the formal maximum of $\Re s$ in (4.17) vanishes. The corresponding wave number

$$
q^{2 /(1-\gamma)} \sim-2 \frac{\Re w_{2}}{\Re w_{1}}
$$

is infinite at $d=d_{O}$. Figures 4 and 5 depict a typical solution reconstruction by series (4.17) up to second order for $d=0.95 d_{O}$ and $d=1.05 d_{O}$ respectively.
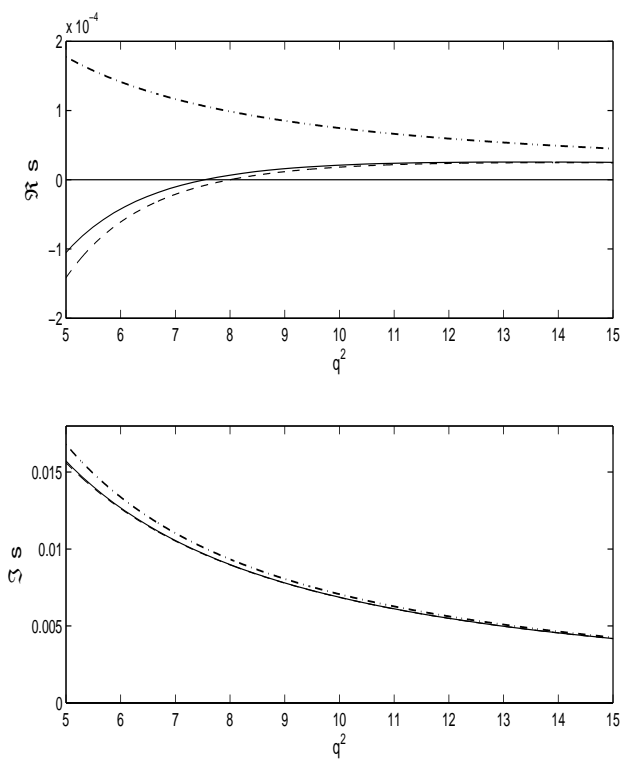

Figure 4: Sub-critical complex branch ( $\operatorname{det} \nabla \mathbf{f}=0.3, \operatorname{tr} \nabla \mathbf{f}=-0.5, \operatorname{tr}_{w} \nabla \mathbf{f}=1.5, \gamma=\frac{1}{5}$, $d=0.95 d_{O}$ ): numerical solution ( solid ), leading order ( dash-dotted ) and second order approximation ( dashed).

Rendering $\operatorname{tr}_{w} \nabla \mathbf{f}$ fixed, by (4.25) the anomalous system is more unstable since $d_{O}>$ $d_{M} \forall \gamma \neq 1$. In physical interpretation, existence of oscillatory unstable modes allows for instability beyond $d_{M}$, the normal critical value. Returning to the conventional approach of fixed values of $\nabla \mathbf{f}$ and letting $\operatorname{tr}_{w} \nabla \mathbf{f}$ vary in accordance with $d,(4.25)$ can be solved as a 

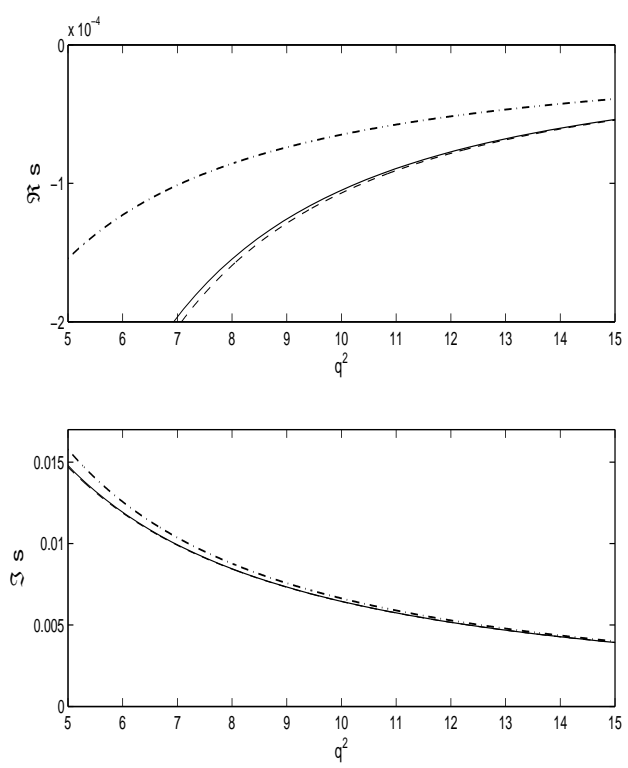

Figure 5: Super-critical complex branch ( $\operatorname{det} \nabla \mathbf{f}=0.3, \operatorname{tr} \nabla \mathbf{f}=-0.5, \operatorname{tr}_{w} \nabla \mathbf{f}=1.5, \gamma=\frac{1}{5}$, $\left.d=1.05 d_{O}\right)$ : numerical solution ( solid ), leading order ( dash-dotted ) and second order approximation ( dashed).

quadratic equation for $d_{O}$ ( choosing the larger root since $d>1$ ):

$$
d_{O}=\frac{2 \operatorname{det} \nabla \mathbf{f} \sin ^{2} \frac{\pi \gamma}{2}-\nabla \mathbf{f}_{11} \nabla \mathbf{f}_{22}}{\nabla \mathbf{f}_{11}^{2}}\left(1+\sqrt{1-\left(\frac{\nabla \mathbf{f}_{11} \nabla \mathbf{f}_{22}}{2 \operatorname{det} \nabla \mathbf{f} \sin ^{2} \frac{\pi \gamma}{2}-\nabla \mathbf{f}_{11} \nabla \mathbf{f}_{22}}\right)^{2}}\right) .
$$

In the case of normal diffusion instability will ensue for the values of $d$ above the normal critical value $d_{M}$ and for anomalous diffusion $d_{O}<d_{M}$, again yielding a more unstable system. Figure 6 shows an example of the anomalous critical ratio versus the exponent $\gamma$. Similar results were obtained numerically in [18].

\section{Stability in the case of different anomaly exponents}

\section{1. $\quad$ General case $\left|\gamma_{1}-\gamma_{2}\right| \sim O(1)$}

Consider the growth rate asymptotics in the short wave region $q \gg 1$. Similarly to the case of equal exponents an expansion in powers of $q$ is used:

$$
s \sim w_{1} q^{\mu_{1}}\left(1+\sum_{j=2}^{\infty} w_{j} q^{\mu_{j}}\right), \quad w_{j} \sim O(1), \quad \mu_{j}<0 \forall j, \quad \mu_{j}<\mu_{j-1} \forall j \geq 2,
$$

only here the exponents $\mu_{j}$ are not multiples of $\mu_{1}$. Substitution of (5.1) into (3.6) and scrutiny of the powers of $q$ reveals that there is no symmetry between $\gamma_{1}$ and $\gamma_{2}$ in the sense 


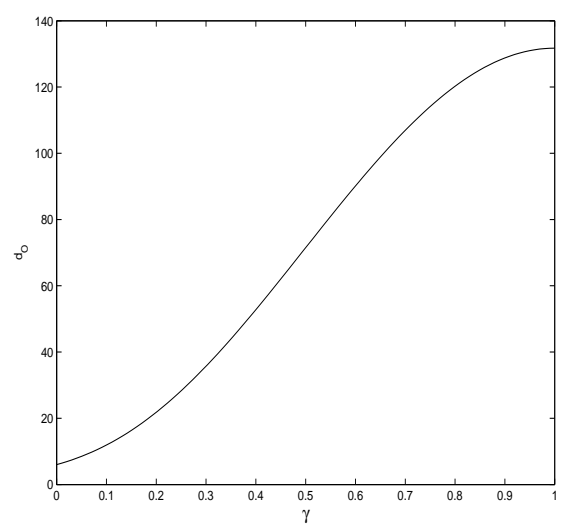

Figure 6: Anomalous critical diffusion coefficients' ratio $d_{O}$ versus anomaly exponent $\gamma$ $\left(\operatorname{det} \nabla \mathbf{f}=0.3, \nabla \mathbf{f}_{11}=0.1, \nabla \mathbf{f}_{22}=-0.6, \operatorname{tr} \nabla \mathbf{f}=-0.5\right)$.

that their interchange results in a different problem. To determine the leading order, first suppose $\gamma_{1}<\gamma_{2}$. Then

$$
\left|s^{1-\gamma_{1}}\right| \ll\left|s^{1-\gamma_{2}}\right| \text { when }|s| \ll 1,
$$

only two terms can be of the same order instead of three for equal anomaly exponents (4.19) and there are two branches. Hereinafter the exponents $\mu_{j k}$ and the functions $w_{j k}$ denote the $j$-th term in series (5.1) of the $k$-th branch, $k \in\{1,2\}$. The leading order terms in (3.6) are

$$
\begin{array}{lr}
O\left(q^{0}\right): & \mu_{11}=-\frac{2}{1-\gamma_{2}}, \quad w_{11}^{1-\gamma 2}=\operatorname{det} \nabla \mathbf{f} /\left(d \nabla \mathbf{f}_{11}\right), \\
O\left(q^{2\left(\gamma_{2}-\gamma_{1}\right) /\left(1-\gamma_{1}\right)}\right): & \mu_{12}=-\frac{2}{1-\gamma_{1}}, \quad w_{12}^{1-\gamma_{1}}=\nabla \mathbf{f}_{11} .
\end{array}
$$

The second balanced orders will be respectively

$$
\begin{array}{cc}
O\left(q^{2\left(\gamma_{1}-\gamma_{2}\right) /\left(1-\gamma_{2}\right)}\right): & \mu_{21}=2 \frac{\gamma_{1}-\gamma_{2}}{1-\gamma_{2}}, \quad w_{21}=\frac{\left(\operatorname{det} \nabla \mathbf{f}-\nabla f_{11} \nabla f_{22}\right) w_{11}^{1-\gamma_{1}}}{\left(1-\gamma_{2}\right) \nabla f_{11} \operatorname{det} \nabla \mathbf{f}}, \\
O\left(q^{0}\right): & \mu_{22}=2 \frac{\gamma_{1}-\gamma_{2}}{1-\gamma_{1},}, w_{22}=\frac{\nabla f_{11} \nabla f_{22}-\operatorname{det} \nabla \mathbf{f}}{d\left(1-\gamma_{1}\right) \nabla f_{11} w_{1}^{1-\gamma_{2}}} .
\end{array}
$$

Similarly, if $\gamma_{1}>\gamma_{2}$,

$$
\begin{aligned}
& O\left(q^{0}\right): \quad \mu_{11}=-\frac{2}{1-\gamma_{1}}, \quad w_{11}^{1-\gamma_{1}}=\operatorname{det} \nabla \mathbf{f} / \nabla \mathbf{f}_{22}, \\
& O\left(q^{2\left(\gamma_{1}-\gamma_{2}\right) /\left(1-\gamma_{2}\right)}\right): \quad \mu_{12}=-\frac{2}{1-\gamma_{2}}, \quad w_{12}^{1-\gamma_{2}}=\nabla \mathbf{f}_{22} / d .
\end{aligned}
$$

The next order terms will be respectively

$$
O\left(q^{2\left(\gamma_{2}-\gamma_{1}\right) /\left(1-\gamma_{1}\right)}: \quad \mu_{21}=2 \frac{\gamma_{2}-\gamma_{1}}{1-\gamma_{1}}, \quad w_{21}=\frac{\left(\operatorname{det} \nabla \mathbf{f}-\nabla f_{11} \nabla f_{22}\right) d w_{11}^{1-\gamma_{2}}}{\left(1-\gamma_{1}\right) \nabla f_{22} \operatorname{det} \nabla \mathbf{f}}\right.
$$




$$
O\left(q^{0}\right): \quad \mu_{22}=2 \frac{\gamma_{2}-\gamma_{1}}{1-\gamma_{2}}, \quad w_{22}=\frac{\nabla f_{11} \nabla f_{22}-\operatorname{det} \nabla \mathbf{f}}{\left(1-\gamma_{2}\right) \nabla f_{22} w_{12}^{1-\gamma_{1}}} .
$$

Scrutiny of the decaying branches $s(q)$ given by (5.3)-(5.6) reveals that the diffusion coefficients' ratio $d$ no longer governs the system stability.

When $\gamma_{1}<\gamma_{2}$, by $(5.3 \mathrm{a}, \mathrm{b})$ the first term of the series (5.1) is real and positive for both branches, i.e. the system is never stable because a real positive root $s$ corresponds to a real positive $\sigma=s^{1 / \mathcal{R}}$, located within the instability sector. The value of $d$ will only determine the growth rate of the unstable modes. This feature ensues because not only is the diffusion coefficients' ratio in favour of the inhibitor, but also the diffusion of the activator is essentially slower ( smaller anomaly exponent). Figure 7 shows a typical solution and polar map. For small values of $q$ there are two complex non-conjugate branches, one of which joins the double real branch at $q=q_{m}$. It is of importance that existence of oscillatory unstable modes is still governed by the value of $d$, however for large enough wave numbers monotonous modes are present, rendering the system unstable.

Figure 8 shows the range of moderate $q$ for different values of diffusion coefficients' ratio. Notice the diminuent growth rate for the larger values of $d$. For wave numbers smaller than the merge point value $q<q_{m}$ an additional complex root exists and it is not shown for the sake of simplicity.
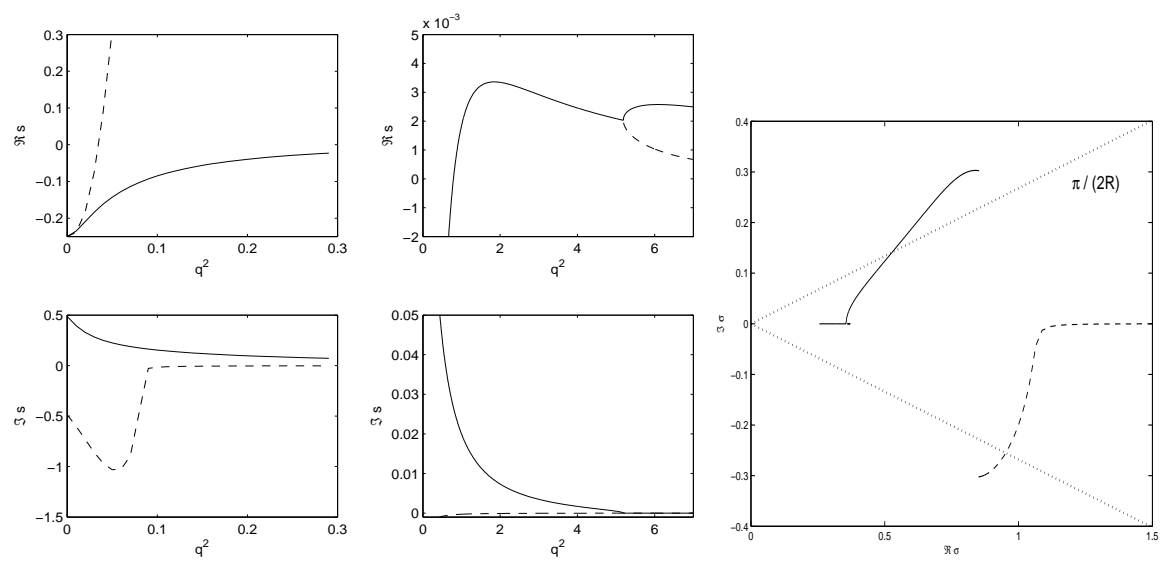

Figure 7: Solution ( left ) and polar map ( right ) for $\gamma_{1}<\gamma_{2}(\operatorname{det} \nabla \mathbf{f}=0.3, \operatorname{tr} \nabla \mathbf{f}=-0.5$, $\left.\operatorname{tr}_{w} \nabla \mathbf{f}=1.5, \gamma_{1}=\frac{1}{6}, \gamma_{2}=\frac{1}{2}, d=23\right)$. The real part of the dashed branch is not shown in whole due to its large magnitude.

When $\gamma_{1}>\gamma_{2}$, by $(5.5 \mathrm{a}, \mathrm{b})$ the first term of (5.1) is complex and the two branches do not form a conjugate pair. Hence stability properties require further analysis via (3.8). The respective argument angles are $\pi /\left[\left(1-\gamma_{j}\right) \mathcal{R}\right], j \in\{1,2\}$, never lying inside the required sector. Therefore neither of these branches is unstable.

In addition to roots that decay for large wave numbers it is possible to seek a non-decaying 

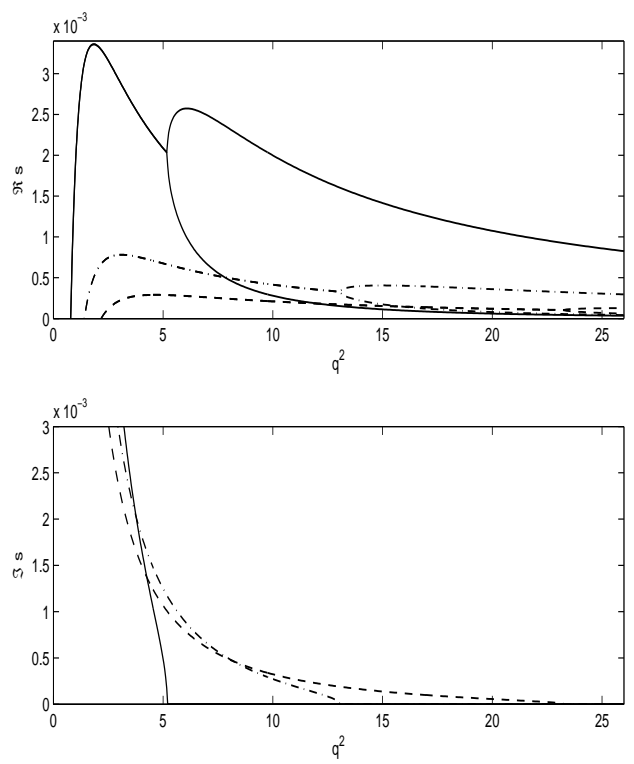

Figure 8: Solution for $\gamma_{1}<\gamma_{2}$ for growing values of $d$ ( $\operatorname{det} \nabla \mathbf{f}=0.3, \operatorname{tr} \nabla \mathbf{f}=-0.5$, $\operatorname{tr}_{w} \nabla \mathbf{f}=1.5, \gamma_{1}=\frac{1}{6}, \gamma_{2}=\frac{1}{2}$ ): $d=23$ ( solid ), $d=44.5$ ( dash-dotted ) and $d=66$ ( dashed ). For $q<q_{m}$ an additional complex root exists, not shown out of simplicity.

branch in the form

$$
s \sim W_{1} q^{\nu_{1}}\left(1+\sum_{j=2}^{\infty} W_{j} q^{\nu_{j}}\right), \quad W_{j} \sim O(1) \forall j, \quad \nu_{1}>0, \quad \nu_{j}<0, \nu_{j}<\nu_{j-1} \forall j \geq 2 .
$$

Substitution into (3.6) and similar steps as in the analysis of decaying roots yields two branches for each case. When $\gamma_{1}<\gamma_{2}$,

$$
\begin{array}{ll}
O\left(q^{4 / \gamma_{1}}\right): & \nu_{11}=\frac{2}{\gamma_{1}}, W_{11}^{\gamma_{1}}=-1, \\
O\left(q^{2+2\left(2-\gamma_{1}\right) / \gamma_{2}}\right): & \nu_{12}=\frac{2}{\gamma_{2}}, \quad W_{12}^{\gamma_{2}}=-d .
\end{array}
$$

For the first branch the terms of order $O\left(q^{2+2\left(2-\gamma_{2}\right) / \gamma_{1}}\right)$ cancel. Then the next balanced order is $O\left(q^{2 / \gamma_{1}}\right)$ :

$$
\nu_{21}=-\frac{2}{\gamma_{1}}, \quad W_{21}=\frac{\nabla f_{11}}{\gamma_{1} W_{11}} .
$$

For the second branch the terms of orders $O\left(q^{4 / \gamma_{2}}\right)$ and $O\left(q^{2 / \gamma_{2}}\right)$ cancel. The next balanced order is $O\left(q^{2+2\left(1-\gamma_{1}\right) / \gamma_{2}}\right)$ :

$$
\nu_{22}=-\frac{2}{\gamma_{2}}, \quad W_{22}=\frac{\nabla f_{22}}{\gamma_{2} W_{12}}
$$


When $\gamma_{1}>\gamma_{2}$, the branches ensue by

$$
O\left(q^{4 / \gamma_{2}}\right): \quad \nu_{11}=\frac{2}{\gamma_{2}}, \quad W_{11}^{\gamma_{2}}=-d
$$

and $O\left(q^{2+2\left(2-\gamma_{2}\right) / \gamma_{1}}\right)$

$$
O\left(q^{2+2\left(2-\gamma_{2}\right) / \gamma_{1}}\right): \quad \nu_{12}=\frac{2}{\gamma_{1}}, \quad W_{12}^{\gamma_{1}}=-1
$$

For the first branch the terms of order $O\left(q^{2+2\left(2-\gamma_{1}\right) / \gamma_{2}}\right)$ cancel. Then the next balanced order is $O\left(q^{2 / \gamma_{2}}\right)$ :

$$
\nu_{21}=-\frac{2}{\gamma_{2}}, \quad W_{21}=\frac{\nabla f_{22}}{\gamma_{2} W_{11}} .
$$

For the second branch the terms of orders $O\left(q^{4 / \gamma_{2}}\right)$ and $O\left(q^{2 / \gamma_{2}}\right)$ cancel. The next balanced order is $O\left(q^{2+2\left(1-\gamma_{2}\right) / \gamma_{1}}\right)$ :

$$
\nu_{22}=-\frac{2}{\gamma_{1}}, \quad W_{22}=\frac{\nabla f_{11}}{\gamma_{1} W_{12}}
$$

It is remarkable that the solutions for $\gamma_{1} \lessgtr \gamma_{2}$ coincide to second order and yet balance terms of 4 orders of magnitude, all different for $\gamma_{1}<\gamma_{2}$ and $\gamma_{1}>\gamma_{2}$. Since the terms cancelling and powers equated are also different, it is impossible to propose that the solutions are identical to arbitrary order.

To determine the stability of (5.10) criterion (3.8) is used with the argument angle being $\pi /\left(\gamma_{1} \mathcal{R}\right)$, again never lying inside the instability sector. Therefore when $\gamma_{1}>\gamma_{2}$, the system is stable. Figure 9 shows a typical solution as a function of $q^{2}$ and polar map. Notice that the roots do not form a conjugate pair. The intermediate range of wave numbers is shown to clarify the behavior of the curves. It must be noted that the asymptotic analysis treats infinitely short waves, and hence presence of oscillatory unstable modes for waves of moderate length is not excluded.

Regarding the monotonous modes, the anomalous problem can be viewed as the following heuristic transformation of the normal one: the diffusion coefficient of the activator, unity in the dimensionless formulation, is replaced by the quantity $s^{1-\gamma_{1}}$ and that of the inhibitor - by $d s^{1-\gamma_{2}}$. Since the instability nature is monotonous, $s$ is real and positive, and thus it is possible to define an effective diffusion ratio

$$
d_{e f f}=d s^{\gamma_{1}-\gamma_{2}} .
$$

Therefore

$$
\lim _{s \rightarrow 0} d_{e f f}= \begin{cases}\infty & \gamma_{1}<\gamma_{2} \\ 0 & \gamma_{1}>\gamma_{2}\end{cases}
$$

Keeping the entries of $\nabla \mathbf{f}$ fixed ( as was tacitly done in the asymptotic analysis above ), the well known results of normal diffusion are recovered: instability ensues for large values of the diffusion coefficients' ratio $d>d_{M}$, whereas for its small values the system is stable $d<d_{M}$. 

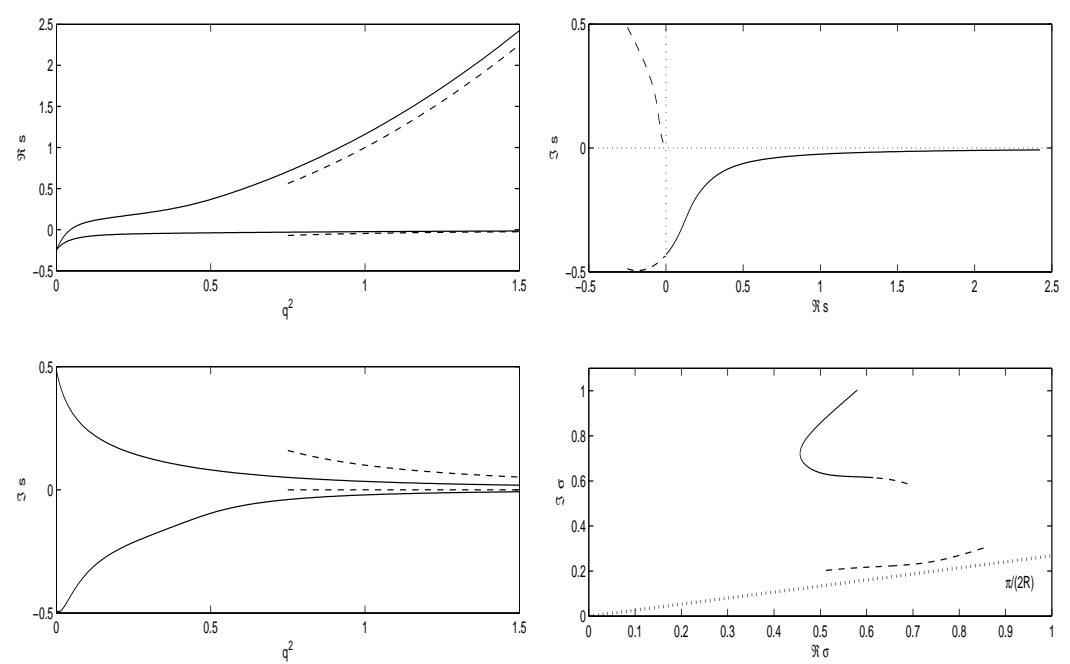

Figure 9: Solution ( left ) and polar map ( right )for $\gamma_{1}>\gamma_{2}(\operatorname{det} \nabla \mathbf{f}=0.3, \operatorname{tr} \nabla \mathbf{f}=-0.5$, $\operatorname{tr}_{w} \nabla \mathbf{f}=1.5, \gamma_{1}=\frac{1}{2}, \gamma_{2}=\frac{1}{6}, d=23$ ): numerical solution ( solid ) and second order asymptotic approximation ( dashed ).

\subsection{The case $\left|\gamma_{2}-\gamma_{1}\right| \ll 1$}

When the anomaly exponents equalled, the main scale of the decaying solutions was $s \sim$ $q^{-2 /(1-\gamma)}$ and the diffusion coefficients' ratio $d$ controlled the onset of instability and its monotonous or oscillatory nature. Monotonous instability also ensued when the exponents differed in favor of the inhibitor $\left(\gamma_{1}<\gamma_{2}\right)$, with each $\gamma_{j}$ dictating its own attenuation scale $s \sim q^{-2 /\left(1-\gamma_{j}\right)}$. Thus it is natural to expect that the case of slightly differing exponents will entail an interesting scale interplay.

As noted before, the dispersion relation (3.6) is not symmetrical in $\gamma_{j}$. Hence, for close exponents linearization both about $\gamma_{1}$ and $\gamma_{2}$ is necessary. Taking

$$
\delta \gamma=\gamma_{2}-\gamma_{1}, \quad|\delta \gamma| \ll 1
$$

expanding (3.6) about $\gamma_{1}$ with respect to $\delta \gamma$ it is possible to seek roots in the form

$$
s=s_{0}+\delta \gamma s_{1}+O\left((\delta \gamma)^{2}\right)
$$

Obviously, the function $s_{0}$ coincides with the solution for $\gamma_{1}=\gamma_{2}$. Bearing in mind that in (3.6) $\gamma_{1}$ appears in power indicators of $s$, the function $s_{1}$ is expected to contain terms of the type $\log s_{0}$, thereby posing the question of proper convergence of (5.15) for fixed $\delta \gamma$. Expressions for $s_{0}, s_{1}$ and proof of convergence of (5.15) is brought in appendix C.

Qualitatively, the growth rate curves $s(q)$ for close exponents resemble figure 7. If $\gamma_{1}<\gamma_{2}$, two pairs of complex conjugate roots start from the known roots at $q=0$. One branch diverges in magnitude as the wave number $q$ grows, the other branch develops moderately and joins the double real branch at $q=q_{m}$. For $q>q_{m}$ both roots are real. If $\gamma_{1}>\gamma_{2}$, there are two complex non-conjugate branches for all values of $q$. 
In the case $\gamma_{1}<\gamma_{2}$ the merging point of the double real branch shifts to infinity similarly to the equal exponents case with $d \nearrow d_{M}$. In physical interpretation, a small difference in anomaly exponents in favor of the inhibitor may be regarded as a sub-critical diffusion coefficients' ratio. Likewise, when $\gamma_{1}>\gamma_{2}$, the decaying curve resembles the stable roots of the equal exponents case with $d \searrow d_{O}$, i.e. a small difference in anomaly exponents in favor of the activator should be viewed as a super-critical diffusion coefficients' ratio. For the infinitely short waves the range $d_{M}<d<d_{O}$ loses relevance when $\gamma_{1} \neq \gamma_{2}$ even if $|\delta \gamma| \ll 1$, since eventually the mean square dispersion of the particles with smaller anomaly exponent will be essentially slower, either giving rise to monotonous instability or subduing the onset of instability at all.

For waves of moderate length oscillatory instability is still possible and its onset depends on the value of $d$. For example, if a system with equal anomaly exponents manifests both oscillatory and monotonous unstable modes for a given value of $d$, a slight increase of the acivator exponent $\gamma_{1}$ will subdue the monotonous instability as both branches turn complex, but the oscillatory modes will remain unstable. Growth rate curves $s(q)$ and a polar map of $\sigma$ in figure 10 exemplify such a transition. The oscillatory instability disappears as the value of $d$ increases ( with $\operatorname{tr}_{w} \nabla \mathbf{f}$ remaining fixed ). Polar map of $\sigma$ in figure 11 depicts a transition from oscillatory instability to neutrality and to a stable system.
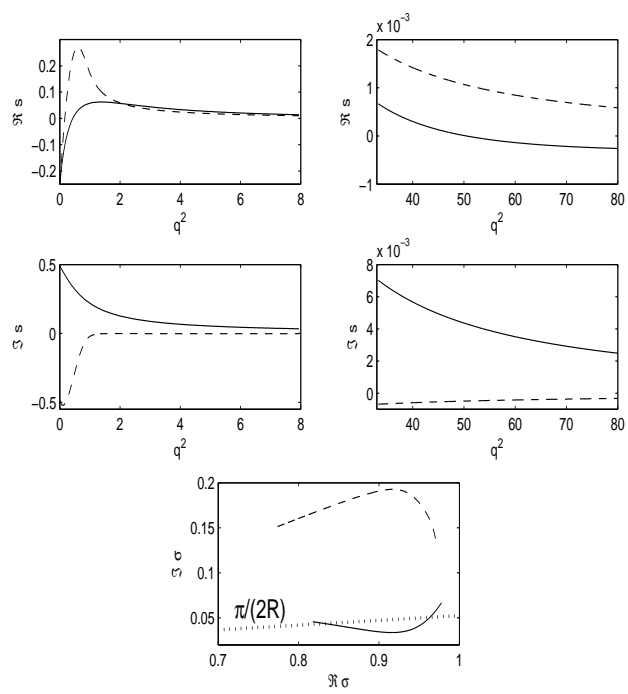

Figure 10: Oscillatory instability of waves of intermediate length for $\gamma_{1}>\gamma_{2}$ ( $\operatorname{det} \nabla \mathbf{f}=0.3$, $\left.\operatorname{tr} \nabla \mathbf{f}=-0.5, \operatorname{tr}_{w} \nabla \mathbf{f}=1.5, \gamma_{1}=\frac{1}{5}, \gamma_{2}=\frac{1}{6}, d=1.4\right)$. Solid and dashed lines denote the two solution branches. Plots of $s$ are split in two ranges of $q$ for clarity. The polar map for $\sigma$ is shown in whole.

Hence, if the difference between the diffusion anomaly exponents is small, the splitting of the power law exponents is absent at the leading order and emerges only as a next-order effect. 


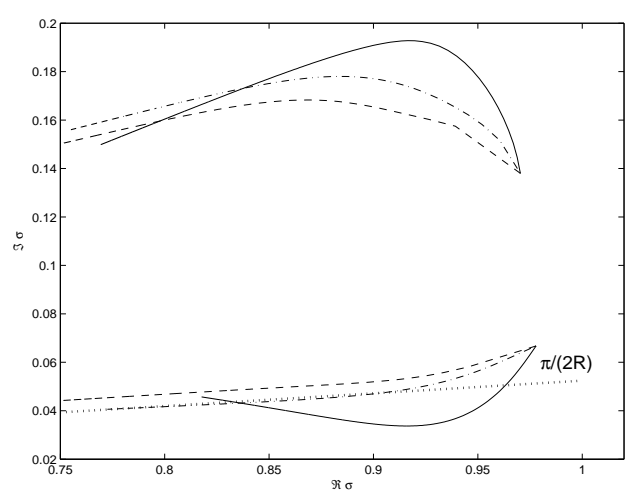

Figure 11: Oscillatory instability of waves of intermediate length for $\gamma_{1}>\gamma_{2}$ - polar map of $\sigma$ for several values of $d\left(\operatorname{det} \nabla \mathbf{f}=0.3, \operatorname{tr} \nabla \mathbf{f}=-0.5, \operatorname{tr}_{w} \nabla \mathbf{f}=1.5, \gamma_{1}=\frac{1}{5}, \gamma_{2}=\frac{1}{6}\right): d=1.4$ ( solid ), $d=14$ ( dash-dotted ) and $d=66$ ( dashed ).

\section{Discussion}

The investigation treated a two species anomalous diffusion system with rotational invariance with general non-linear kinetics. Derived conditions for Turing instability revealed an anomaly dependent restriction, i.e. in lieu of the requirement of roots' location in the right open half plane, well known from normal diffusion, in anomalous systems the roots of the dispersion relation, transformed into polynomial form, must lie within a smaller sector $(-\pi /(2 \mathcal{R}), \pi /(2 \mathcal{R}))$.

When the anomaly exponents equal, for activator-inhibitor diffusion coefficients' ratio below the normal critical value $d<d_{M}$ the growth rate curve as a function of the wave number $s(q)$ possesses two real branches for $q>q_{m}$ and a complex branch for $0<q<q_{m}$. The double real branch transduces from the positive range of the analogous curve in the case of normal diffusion. The complex branch starts at the stable point $\left(0,\left.s\right|_{q=0}\right)$, crosses the real line at $0<q_{0}<q_{m}$ ( pure imaginary root ) and joins the real branches at $\left(q_{m}, s_{m}\right)$, their merge point. As long as $d<d_{M}$, the real branches give monotonously divergent modes, and the range of wave numbers $0<q_{0}<q<q_{m}$ with $s \in \mathbb{C}$ gives unstable modes of oscillatory nature.

As the inhibitor-activator diffusion coefficients' ratio exceeds the normal critical value $d>$ $d_{M}, q_{m}$ shifts to infinity and the real branch merges into the real line. For normal diffusion no instability is then exhibited. In an anomalous system with fractional derivative acting on both reaction and diffusion terms the only unstable modes are monotonous, disappearing at the threshold $d_{M}$ identically to a normal system $[12,13]$. Conversely, with diffusion anomaly accompanied by normal reaction, the range $q>q_{0}$ remains unstable. The growth rate curve $s(q)$ possesses a local maximum, therefore for a fixed value of $d$ the infinitely short waves are never dominant. The curve decay with the wave number $q$ obeys a power law with the exponent $-2 /(1-\gamma)$. 
There exists a ratio $d_{O}>d_{M}$, above which no instability is observed, i.e.

$$
\Re s \searrow 0 \text { as } q \rightarrow \infty, d_{M}<d<d_{O}
$$

and

$$
\Re s \nearrow 0 \text { as } q \rightarrow \infty, d>d_{O} .
$$

The expression for $d_{O}$, first derived as a leading order approximation, turned out to be correct to arbitrary order and thus exact.

When the anomaly exponents differ, at the infinitely short waves limit the curves $s(q)$ develop according to the scales $-2 /\left(1-\gamma_{j}\right)$ for decaying branches and $2 / \gamma_{j}$ for diverging ones. The distinct anomaly exponents govern the onset of instability, whereas the ratio of diffusion coefficients affects the complex growth rate only. When $\gamma_{1}<\gamma_{2}$, i.e. the activator diffusion is more anomalous, the system is always unstable, whereas when $\gamma_{1}>\gamma_{2}$, no monotonous instability is observed. This theoretical conclusion generalizes the result long known for normal diffusion: faster diffusion of the inhibitor, expressed via the coefficients' ratio $d$, is necessary for the onset of instability. For anomalous diffusion distinct exponents set the diffusion scales so far apart that the ratio $d$ becomes meaningless.

It may be heuristically replaced by an effective ratio $d_{e f f}=d s^{\gamma_{1}-\gamma_{2}}$, recovering again the results for normal diffusion: when $|s| \rightarrow 0$,

$$
d_{\text {eff }} \rightarrow 0 \text { for } \gamma_{2}<\gamma_{1}
$$

corresponds to stable values $d \ll 1$ ( in particular $d<d_{M}$ ), whereas

$$
d_{e f f} \rightarrow \infty \text { for } \gamma_{2}>\gamma_{1}
$$

corresponds to unstable values $d \gg 1\left(d>d_{M}\right)$.

To complete the picture, in the case of slower anomalous diffusion for the activator semiinfinite range of short ustable waves $q>q_{m}$ exists, similarly to the equal anomaly exponents case with $d<d_{M}$ ( with $\operatorname{tr}_{w} \nabla \mathbf{f}$ fixed ). When the diffusion of the inhibitor is slower, the short waves are stable, yet waves of moderate length may exhibit oscillatory instability, depending of the diffusion coefficients' ratio $d$. The existence of such unstable modes $(s \in \mathbb{C})$ is possible, as the performed asymptotic analysis was limited to infinitely short waves. A summary of these properties is brought in table 1.

When the difference between the anomaly exponents is small, the attenuating growth rate curves may be approximated by a Taylor expansion in $\delta \gamma=\gamma_{2}-\gamma_{1}$ about the equal exponents solution. It was shown that the expansion converged properly up to order $O(|\delta \gamma|)$. Regarding instability, there is no qualitative difference between a system with distinct anomaly exponents and infinitesimally close ones.

The main innovation of the current work is the exploration of oscillatory ( complex ) modes of the system, bearing generalized conditions for Turing instability, anomalous critical ratio $d_{O}$ for equal exponents and scale separation for distinct exponents. For equal exponents and sub-critical $d$ the oscillatory unstable modes coexist with the monotonous ones. The 


\begin{tabular}{|c|c|c|c|}
\hline \hline exponents & $d$ & monotonous instability & oscillatory instability \\
\hline$\gamma_{1}=\gamma_{2}=\gamma$ & $d>d_{M}$ & $q>q_{m}$ & $q_{0}<q<q_{m}$ \\
\hline$\gamma_{1}=\gamma_{2}=\gamma$ & $d_{O}<d<d_{M}$ & none & $q>q_{0}$ \\
\hline$\gamma_{1}<\gamma_{2}$ & $d>1$ & $q>q_{m}$ & $q_{0}<q<q_{m}$ \\
\hline$\gamma_{1}>\gamma_{2}$ & - & none, $d>1$ & $q \sim O(1), d-$ dependent \\
\hline \hline
\end{tabular}

Table 1: Stability properties for different combinations of anomaly exponents ( with fixed entries of $\nabla \mathbf{f}$ ).

oscillatory instability is retained beyond the critical point $d=d_{M}$ for a $\gamma$-dependent set of $d$. Moreover, complex modes allow for instability at intermediate wave numbers even when the activator anomaly exponent exceeds that of the inhibitor. The unusual choice of the sensitivity matrix weighted $\operatorname{trace} \operatorname{tr}_{w} \nabla \mathbf{f}=d \nabla \mathbf{f}_{11}+\nabla \mathbf{f}_{22}$ as a fixed parameter kept all derivations analytical. Upon appropriate parameter adjustment these results correspond to numerical studies $[16,18]$.

Living cells might be an excellent example for a system with anomalous diffusion ( due to the intrinsic properties of the endoplasmic reticulum ) and simultaneously normal reactions with components of abundant concentration: molecules transport through the cell might be hindered, but the reaction will occur normally once the particular molecule got to its destination. For instance, a protein that is supposed to cross a membrane of an organelle will diffuse anomalously through the endoplasmic reticulum, but be binded normally to the correct receptor on the membrane. In the past decade experiments designed to observe and measure anomalous diffusion in living cells showed that many diffusive processes traditionally modelled as normal were actually anomalous [20]. Observation of instability and pattern formation might be expected in these systems upon further amelioration in experimental techniques. Another interesting example is a gel medium. There separate experiments revealed instability ( in particular Turing patterns ) [21] and sub-diffusion [3]. Thus one can expect observations of Turing instability in sub-diffusive gel solvents.

From the mathematical standpoint a non-linear instability theory is of interest for future research.

Acknowledgements. The support of B. and G. Greenberg Research Fund ( Ottawa), Minerva Center for Nonlinear Physics of Complex Systems and Israel Science Foundation ( grant \# 812/06 ) is acknowledged. 


\section{Appendix A. Instability sector}

For $\gamma_{1}=\gamma_{2}=\gamma$ in the Fourier domain the perturbed density vector will have the form

$$
\begin{gathered}
\widehat{\Delta \mathbf{n}}(q, t)=\frac{1}{2 \pi i} \int_{c-\imath \infty}^{c+i \infty} I(q, s) e^{s t} d s, \quad I(q, s)=\frac{1}{D(q, s ; \gamma)}\left(\mathcal{A}(q) s+\mathcal{B}(q) s^{1-\gamma}+\mathcal{C}(q)\right) \\
\mathcal{A}(q)=\left.\widehat{\Delta \mathbf{n}}\right|_{t=0}, \quad \mathcal{B}_{j}(q)=\left.q^{2} C_{k k} \widehat{\Delta n_{j}}\right|_{t=0}, \quad \mathcal{C}_{j}(q)=\left.\nabla \mathbf{f}_{j k} \widehat{\Delta n_{k}}\right|_{t=0}-\left.\nabla \mathbf{f}_{k k} \widehat{\Delta n_{j}}\right|_{t=0} \\
j, k \in\{1,2\}, j \neq k,
\end{gathered}
$$

and $c \in \mathbb{R}$ lying to the right of all integrand singularities.

Temporal evolution of $\widehat{\Delta \mathbf{n}}$ follows via the residue theorem:

$$
\begin{gathered}
\widehat{\Delta \mathbf{n}}(q, t)=\lim _{R \rightarrow \infty} \frac{1}{2 \pi i} \int_{F A} I(q, s) e^{s t} d s=\frac{1}{2 \pi i}\left(\lim _{\epsilon \rightarrow 0, R \rightarrow \infty} \oint_{\Gamma} I(q, s) e^{s t} d s-\right. \\
\lim _{R \rightarrow \infty} \int_{A B} I(q, s) e^{s t} d s-\lim _{\epsilon \rightarrow 0, R \rightarrow \infty} \int_{B C} I(q, s) e^{s t} d s-\lim _{\epsilon \rightarrow 0} \int_{C D} I(q, s) e^{s t} d s- \\
\left.\lim _{\epsilon \rightarrow 0, R \rightarrow \infty} \int_{D E} I(q, s) e^{s t} d s-\lim _{R \rightarrow \infty} \int_{E F} I(q, s) e^{s t} d s\right) .
\end{gathered}
$$

All integration paths are shown in figure 12. By immediate generalization of a similar integration procedure in [16], the integrals along all arcs vanish in the corresponding limits and the integrals along the branch cut attenuate in time. The closed contour integral remains the only possible term to entail instability.

Without loss of generality $\gamma$ is taken to be a simple reduced fraction $\gamma=m / r$, as the set of rational numbers is dense within the set of real numbers, and a new integration variable is introduced

$$
\sigma=s^{1 / r}=|s|^{1 / r} e^{i \arg (s) / r} .
$$

For correct Laplace transform inversion the main branch was chosen, preventing contour rotation and consequent inclination of the line $F A$. Obviously, the shape of $\Gamma$ is modified from a circle-like to a sector-like according to the value of $r$ ( see figure 12 ). The constant $c$ should be prescribed so that the point $c_{\sigma}=c^{1 / r}$ would also lie to the right of all singularities.

The integrand will then have the form

$$
\begin{gathered}
I(q, \sigma)=\frac{1}{D(q, \sigma ; \gamma)}\left(\mathcal{A}(q) \sigma^{r}+\mathcal{B}(q) \sigma^{r-m}+\mathcal{C}(q)\right) \\
D(q, \sigma ; \gamma)=\sigma^{2 r}+(1+d) q^{2} \sigma^{2 r-m}-\operatorname{tr} \nabla \mathbf{f} \sigma^{r}+d q^{4} \sigma^{2(r-m)}-q^{2} \operatorname{tr}_{w} \nabla \mathbf{f} \sigma^{r-m}+\operatorname{det} \nabla \mathbf{f}
\end{gathered}
$$

whence $D(q, \sigma ; \gamma)$ possesses $2 r$ roots.

$$
2 \pi i \widehat{\Delta \mathbf{n}}(q, t)=\lim _{\epsilon \rightarrow 0, R \rightarrow \infty} \oint_{\Gamma_{\sigma}} I(q, \sigma) e^{\sigma^{r} t} r \sigma^{r-1} d \sigma=
$$




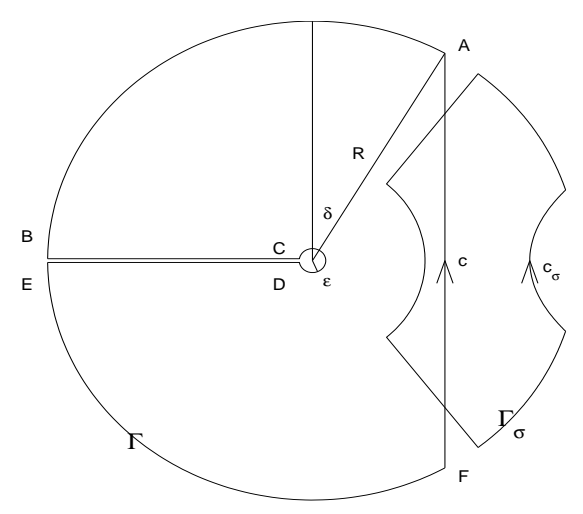

Figure 12: Original $\Gamma$ and modified $\Gamma_{\sigma}$ closed contours for residue computation

$$
\sum_{j=1}^{2 r} \operatorname{Res}\left(I(q, \sigma) e^{\sigma^{r} t} r \sigma^{r-1}\right)=\sum_{j=1}^{r_{\sigma}} \frac{\mathcal{A}(q) \sigma_{j}^{r}+\mathcal{B}(q) \sigma_{j}^{r-m}+\mathcal{C}(q)}{\prod_{k=1, k \neq j}^{2 r}\left(\sigma_{j}-\sigma_{k}\right)} e^{\sigma_{j}^{r} t} r \sigma_{j}^{r-1},
$$

with $r_{\sigma} \leq 2 r$ being the number of singular points $\sigma_{j}$ lying within $\Gamma_{\sigma}$. Thus temporal growth will ensue if at least one point satisfies

$$
\begin{aligned}
& \text { (i) } \Re \sigma^{r}>0 \Leftrightarrow \Re s>0 \\
& \text { (ii) }-\frac{\pi}{r}<\arg \sigma<\frac{\pi}{r} \Leftrightarrow \quad-\frac{\pi}{r}<\arg s^{1 / r}<\frac{\pi}{r},
\end{aligned}
$$

or

$$
-\frac{\pi}{2 r}<\arg s^{1 / r}<\frac{\pi}{2 r}
$$

Note that $m$ determines the number of roots and $r$ - the sector angle.

When the anomaly exponents differ, by (2.8)

$$
\begin{aligned}
D\left(q, s ; \gamma_{1}, \gamma_{2}\right)= & s^{2}+d q^{2} s^{2-\gamma_{2}}+q^{2} s^{2-\gamma_{1}}-\operatorname{tr} \nabla \mathbf{f} s+d q^{4} s^{2-\gamma_{1}-\gamma_{2}}- \\
& d \nabla \mathbf{f}_{11} q^{2} s^{1-\gamma_{2}}-\nabla \mathbf{f}_{22} q^{2} s^{1-\gamma_{1}}+\operatorname{det} \nabla \mathbf{f} .
\end{aligned}
$$

Again, taking $\gamma_{j}=m_{j} / r_{j}, j \in\{1,2\}$ to be reduced fractions, the transformation variable is

$$
\sigma=s^{1 / \mathcal{R}}, \quad \mathcal{R}= \begin{cases}r_{1} & r_{1} / r_{2} \in \mathbb{N} \\ r_{2} & r_{2} / r_{1} \in \mathbb{N} \\ r_{1} r_{2} & \text { otherwise }\end{cases}
$$

and $D\left(q, \sigma ; \gamma_{1}, \gamma_{2}\right)$ possesses $2 \mathcal{R}$ roots. The integrand will take the form

$$
I(q, \sigma)=\frac{1}{D\left(q, \sigma ; \gamma_{1}, \gamma_{2}\right)}\left(\mathcal{A}(q) \sigma^{\mathcal{R}}+\mathcal{B}(q) \sigma^{\mathcal{P}}+\mathcal{C}(q)\right)
$$


where

$$
\mathcal{P}=\left\{\begin{array}{l}
r_{1} / r_{2} \in \mathbb{N} \begin{cases}\left(r_{2}-m_{2}\right) r_{1} / r_{2} & i=1 \\
r_{1}-m_{1} & i=2\end{cases} \\
r_{2} / r_{1} \in \mathbb{N} \begin{cases}r_{2}-m_{2} \\
\left(r_{1}-m_{1}\right) r_{2} / r_{1} & i=2\end{cases} \\
\text { otherwise } \begin{cases}\left(r_{2}-m_{2}\right) r_{1} & i=1 \\
\left(r_{1}-m_{1}\right) r_{2} & i=2 .\end{cases}
\end{array}\right.
$$

Consequently, the instability condition becomes

$$
-\frac{\pi}{2 \mathcal{R}}<\arg s^{1 / \mathcal{R}}<\frac{\pi}{2 \mathcal{R}}
$$

\section{Appendix B. Complex branch in the case of equal anomaly exponents}

Instability of disturbance modes with the wave number in the range $\left(q_{0}, q_{m}\right)$ is inferred by asymptotic analysis of the long wave limit $|q| \ll 1$. To allow for precise argument, it will be assumed henceforth $\Delta_{q=0}<0$ ( see (4.14) for definition ).

Substituting $q_{s}^{2}=s^{1-\gamma} q^{2}$ into (4.8) and linearizing for $q^{2} \ll 1$,

$$
\begin{gathered}
s \sim \alpha+\beta q^{2} s^{1-\gamma}+O\left(q^{4}\right), \\
\alpha:=\left.s\right|_{q=0}, \quad \arg \alpha \in\left[\frac{\pi}{2}, \pi\right] \\
\beta:=\frac{1}{2}\left(-(1+d)+\frac{2 \operatorname{tr}_{w} \nabla \mathbf{f}-(1+d) \operatorname{tr} \nabla \mathbf{f}}{\sqrt{\Delta_{q=0}}}\right), \quad \arg \beta \in\left[\pi, \frac{3 \pi}{2}\right] .
\end{gathered}
$$

$\left.s\right|_{q=0}=\alpha$ enables further linearization of (B.1a) with $|s-\alpha| \ll 1$ :

$$
s \sim \alpha+\frac{\alpha \beta q^{2}}{\alpha^{\gamma}-(1-\gamma) \beta q^{2}}+O\left(q^{4},|s-\alpha|^{2}\right) .
$$

For a fractional power of complex entities the principal branch is taken throughout. Also, for simplicity the error order is omitted hereinafter. Obviously, the approximation is better for smaller values of $\gamma$, when the function $s^{1-\gamma}$ is closer to linear. Extracting the real part,

$$
\Re s \sim \Re \alpha+\frac{n_{4} q^{4}+n_{2} q^{2}}{d_{4} q^{4}+d_{2} q^{2}+d_{0}}
$$




$$
\begin{aligned}
& n_{4}=-(1-\gamma) \Re \alpha|\beta|^{2}>0, \\
& n_{2}=|\alpha|^{1+\gamma}|\beta| \cos (\arg \beta+(1-\gamma) \arg \alpha), \\
& d_{4}=(1-\gamma)^{2}|\beta|^{2}>0, \\
& d_{2}=-2(1-\gamma)|\alpha|^{\gamma}|\beta| \cos (\arg \beta-\gamma \arg \alpha), \\
& d_{0}=|\alpha|^{2 \gamma}>0 .
\end{aligned}
$$

To determine the sign of $d_{2}$, note that for $\operatorname{tr} \nabla \mathbf{f} \nearrow 0$

$$
\arg \alpha \searrow \frac{\pi}{2}, \quad \arg \beta \searrow \arctan \frac{2 \sqrt{d_{M}}}{1+d} \in\left(\pi, \frac{3}{2} \pi\right)
$$

and for $\operatorname{tr} \nabla \mathbf{f} \searrow-2 \sqrt{\operatorname{det} \nabla \mathbf{f}}$

$$
\arg \alpha \nearrow \pi, \quad \arg \beta \nearrow \frac{3}{2} \pi
$$

Hence

$$
\arg \beta-\gamma \arg \alpha>\frac{\pi}{2}
$$

and $d_{2}>0$. As to $n_{2}$, it changes sign for some $0<\gamma<1$ :

$$
\cos (\arg \beta+(1-\gamma) \arg \alpha)=0, \quad \gamma=1-\frac{1.5 \pi-\arg \beta}{\arg \alpha} .
$$

$n_{2}>0$ for smaller values of $\gamma$, where $\arg \alpha \searrow \frac{\pi}{2}$.

Solving $\Re s=0$ yields

$$
\begin{gathered}
q_{0}^{2} \sim \frac{1}{2}\left(-\left(n_{2}+\Re a d_{2}\right)+\sqrt{\Delta_{q_{0}}}\right) /\left(n_{4}+\Re a d_{4}\right) \\
\Delta_{q_{0}}=\left(n_{2}+\Re a d_{2}\right)^{2}-4 \Re a d_{0}\left(n_{4}+\Re a d_{4}\right),
\end{gathered}
$$

where the positive root was chosen due to

$$
n_{4}+\Re \alpha d_{4}=-\Re \alpha|\beta|^{2} \gamma(1-\gamma)>0
$$

and regardless of the sign of $n_{2}+\Re \alpha d_{2}$. Though, if $n_{2}+\Re \alpha d_{2}<0, q_{0}^{2}$ will be too large to satisfy the original assumption $q^{2} \ll 1$. Combining all of the above, for small enough $\gamma$ ( by all means not infinitesimal ) $n_{2}>0, n_{2}+\Re \alpha d_{2}>0$ and consistently $q_{0}^{2} \ll 1$.

Seeking an extremum $\frac{d \Re s}{d q^{2}}=0$ yields

$$
\left(n_{2} d_{4}-n_{4} d_{2}\right) q_{e x t}^{4}-2 d_{0} n_{4} q_{e x t}^{2}-d_{0} n_{2} \sim 0
$$

which after algebraic manipulation is simplified to

$$
\Re s_{\text {ext }}-\Re \alpha \sim \frac{n_{2} q_{e x t}^{2}}{d_{2} q_{e x t}^{2}+2 d_{0}} .
$$


As the denominator is positive, $n_{2}>0$ implies that the curve $\Re s$ ascends $\left(\Re s_{\text {ext }}-\Re \alpha>0\right.$ ) and attains a maximum.

To find out whether the wave numbers $q_{0}<q<q_{m}$ indeed entail instability, it is necessary to perform a consistent linearization for $\sigma$. Omitting the error order,

$$
\begin{aligned}
\sigma & \sim \alpha^{1 / r} \frac{1-\left(1-\gamma-\frac{1}{r}\right) \widetilde{q^{2}}}{1-(1-\gamma) \widetilde{q^{2}}}, \\
\widetilde{q^{2}}:=\beta \alpha^{-\gamma} q^{2} & =|\beta||\alpha|^{\gamma} q^{2} \exp [i(\arg \beta-\gamma \arg \alpha)], \\
\widetilde{q^{2}} & :=\widetilde{q_{r}}+i \widetilde{q_{i}}, \quad q_{r}, q_{i} \in \mathbb{R} .
\end{aligned}
$$

Recalling (B.1b),

$$
\frac{\pi}{2 r} \leq \arg \alpha^{1 / r} \leq \frac{\pi}{r}
$$

and after some algebra

$$
\tan \arg \frac{1-\left(1-\gamma-\frac{1}{r}\right) \widetilde{q^{2}}}{1-(1-\gamma) \widetilde{q^{2}}}=\frac{\widetilde{q_{i}} / r}{1-\left(2-2 \gamma-\frac{1}{r}\right) \widetilde{q_{r}}+(1-\gamma)\left(1-\gamma-\frac{1}{r}\right)\left|\widetilde{q^{2}}\right|^{2}}
$$

The denominator is positive and of unity order within the supposed parameter ranges. The numerator is of order of $q^{2}$, and by (B.1c) there exists a value of $\gamma$, below which $\widetilde{q}_{i}<0$. Hence

$$
|\arg \sigma|<\frac{\pi}{r}
$$

which proves that disturbances with the wave numbers $q_{0}<q<q_{m}$ are linearly unstable when

$$
\Delta_{q=0}<0, \quad 0<\gamma<\min \left\{\left.\gamma\right|_{n_{2}=0},\left.\gamma\right|_{\widetilde{q}_{i}=0}\right\} .
$$

Recalling the limitations imposed on the parameters' values in the course of this derivation, condition (B.15) is sufficient, yet not necessary for the onset of instability.

\section{Appendix C. Solution approximation for close exponents}

Linearizing (3.6) about $\gamma_{1}$ with

$$
\delta \gamma=\gamma_{2}-\gamma_{1}, \quad|\delta \gamma| \ll 1
$$

and seeking roots in the form

$$
s=s_{0}+\delta \gamma s_{1}+O\left((\delta \gamma)^{2}\right)
$$

to leading order gives the equal $\gamma$ equation (3.2) with $\gamma=\gamma_{1}$

$$
D\left(q, s_{0}, \gamma_{1}\right)=0
$$


Hereinafter subscript $\gamma_{j}$ denotes terms resulting from linearization about the equal anomaly exponents problem with $\gamma=\gamma_{j}, j \in\{1,2\}$. The first correction is

$$
\begin{gathered}
\left(s_{1}\right)_{\gamma_{1}}=\log \left(s_{0}\right)_{\gamma_{1}} S_{\text {num }, \gamma_{1}} / S_{\text {den }, \gamma_{1}}, \\
S_{\text {num }, \gamma_{1}}=d q^{2}\left(s_{0}\right)_{\gamma_{1}}^{1-\gamma_{1}}\left(q^{2}\left(s_{0}\right)_{\gamma_{1}}^{1-\gamma_{1}}+s_{0}-\nabla \mathbf{f}_{11}\right), \\
S_{d e n, \gamma_{1}}=2\left(1-\gamma_{1}\right) d q^{4}\left(s_{0}\right)_{\gamma_{1}}^{1-2 \gamma_{1}}+\left(2-\gamma_{1}\right)(1+d) q^{2}\left(s_{0}\right)_{\gamma_{1}}^{1-\gamma_{1}}- \\
\left(1-\gamma_{1}\right) \operatorname{tr}_{w} \nabla \mathbf{f} q^{2}\left(s_{0}\right)_{\gamma_{1}}^{-\gamma_{1}}+2\left(s_{0}\right)_{\gamma_{1}}-\operatorname{tr} \nabla \mathbf{f},
\end{gathered}
$$

or after algebraic manipulation

$$
\begin{gathered}
\left(s_{1}\right)_{\gamma_{1}}=-\left(s_{0}\right)_{\gamma_{1}} \log \left(s_{0}\right)_{\gamma_{1}} \tilde{S}_{n u m, \gamma_{1}} / \tilde{S}_{d e n, \gamma_{1}}, \\
\tilde{S}_{n u m, \gamma_{1}}=q^{2}\left(s_{0}\right)_{\gamma_{1}}^{2-\gamma_{1}}-\nabla \mathbf{f}_{22} q^{2}\left(s_{0}\right)_{\gamma_{1}}^{1-\gamma 1}+\left(s_{0}\right)_{\gamma_{1}}^{2}-\operatorname{tr} \nabla \mathbf{f}\left(s_{0}\right)_{\gamma_{1}}+\operatorname{det} \nabla \mathbf{f}, \\
\tilde{S}_{\text {den }, \gamma_{1}}=\gamma_{1}(1+d) q^{2}\left(s_{0}\right)_{\gamma_{1}}^{2-\gamma_{1}}+\left(1-\gamma_{1}\right) \operatorname{tr}_{w} \nabla \mathbf{f} q^{2}\left(s_{0}\right)_{\gamma_{1}}^{1-\gamma 1}+2 \gamma_{1}\left(s_{0}\right)_{\gamma_{1}}^{2}+ \\
\left(1-2 \gamma_{1}\right) \operatorname{tr} \nabla \mathbf{f}\left(s_{0}\right)_{\gamma_{1}}-2\left(1-\gamma_{1}\right) \operatorname{det} \nabla \mathbf{f} .
\end{gathered}
$$

It should be noted that the expression in the denominator of (C.4) coincides with the derivative of (C.3) under the constraint $d q / d s_{0}=0$, i.e. $s_{1}$ diverges at the merging point of the real branches of $s_{0}$, so the expansion (C.2) is not valid in its vicinity.

Ostensibly, the correction term magnitude $\left|s_{1} / s_{0}\right|$ diverges logarithmically for a fixed value of $\delta \gamma$. Below it will be shown that the factor $S_{\text {num }}$ attenuates fast enough, and no divergence of the series (C.2) is observed as $s_{0}$ decays. For that purpose linearization about $\gamma_{2}$ is necessary. Following similar steps, the first correction becomes

$$
\begin{gathered}
\left(s_{1}\right)_{\gamma_{2}}=\log \left(s_{0}\right)_{\gamma_{2}} S_{\text {num }, \gamma_{2}} / S_{\text {den }, \gamma_{2}}, \\
S_{\text {num }, \gamma_{2}}=q^{2}\left(s_{0}\right)_{\gamma_{2}}^{1-\gamma_{2}}\left[d q^{2}\left(s_{0}\right)_{\gamma_{2}}^{1-\gamma_{2}}+\left(s_{0}\right)_{\gamma_{2}}-\nabla \mathbf{f}_{22}\right], \\
S_{d e n, \gamma_{2}}=2\left(1-\gamma_{2}\right) d q^{4}\left(s_{0}\right)_{\gamma_{2}}^{1-2 \gamma_{2}}+\left(2-\gamma_{2}\right)(1+d) q^{2}\left(s_{0}\right)_{\gamma_{2}}^{1-\gamma_{2}}- \\
\left(1-\gamma_{2}\right) \operatorname{tr}_{w} \nabla \mathbf{f} q^{2}\left(s_{0}\right)_{\gamma_{2}}^{-\gamma_{2}}+2\left(s_{0}\right)_{\gamma_{2}}-\operatorname{tr} \nabla \mathbf{f},
\end{gathered}
$$

or after some algebra

$$
\begin{gathered}
\left(s_{1}\right)_{\gamma_{2}}=-\left(s_{0}\right)_{\gamma_{2}} \log \left(s_{0}\right)_{\gamma_{2}} \tilde{S}_{n u m, \gamma_{2}} / \tilde{S}_{d e n, \gamma_{2}} \\
\tilde{S}_{n u m, \gamma_{2}}=d q^{2}\left(s_{0}\right)_{\gamma_{2}}^{2-\gamma_{2}}-d \nabla \mathbf{f}_{11} q^{2}\left(s_{0}\right)_{\gamma_{2}}^{1-\gamma 2}+\left(s_{0}\right)_{\gamma_{2}}^{2}-\operatorname{tr} \nabla \mathbf{f}\left(s_{0}\right)_{\gamma_{2}}+\operatorname{det} \nabla \mathbf{f} \\
\tilde{S}_{d e n, \gamma_{2}}=\gamma_{2}(1+d) q^{2}\left(s_{0}\right)_{\gamma_{2}}^{2-\gamma_{2}}+\left(1-\gamma_{2}\right) \operatorname{tr}_{w} \nabla \mathbf{f} q^{2}\left(s_{0}\right)_{\gamma_{2}}^{1-\gamma 2}+2 \gamma_{2}\left(s_{0}\right)_{\gamma_{2}}^{2}+ \\
\left(1-2 \gamma_{2}\right) \operatorname{tr} \nabla \mathbf{f}\left(s_{0}\right)_{\gamma_{2}}-2\left(1-\gamma_{2}\right) \operatorname{det} \nabla \mathbf{f}
\end{gathered}
$$

Now suppose $\delta \gamma$ is fixed. When $\left(s_{0}\right)_{\gamma_{1}}$ grows small enough,

$$
\begin{aligned}
& \left|s^{1-\gamma_{1}}\right| \gg\left|s^{1-\gamma_{2}}\right| \text { when }|s| \ll 1, \quad \gamma_{1}>\gamma_{2} \\
& \left|s^{1-\gamma_{2}}\right| \gg\left|s^{1-\gamma_{1}}\right| \text { when }|s| \ll 1, \quad \gamma_{1}<\gamma_{2},
\end{aligned}
$$


i.e. eventually the equations for different exponents will hold. First, take the first branch for the case $\gamma_{1}>\gamma_{2}$. Identifying

$$
q^{2}\left(s_{0}\right)_{\gamma_{1}}^{1-\gamma_{1}} \mapsto w_{11}^{1-\gamma_{1}}
$$

and inserting (5.5a) into the expression for $\tilde{S}_{n u m, \gamma_{1}}$ (C.5),

$$
\left(s_{1}\right)_{\gamma_{1}}=-\left(s_{0}\right)_{\gamma_{1}}^{2} \log \left(s_{0}\right)_{\gamma_{1}} \mathcal{S}_{\gamma_{1}}, \quad \mathcal{S}_{\gamma_{1}} \sim O(1)
$$

For the second branch the scaling involves $\gamma_{2}$, and now

$$
q^{2}\left(s_{0}\right)_{\gamma_{2}}^{1-\gamma_{2}} \mapsto w_{12}^{1-\gamma_{2}}
$$

Inserting (5.5b) into the expression for $S_{n u m, \gamma_{2}}$ (C.6),

$$
\left(s_{1}\right)_{\gamma_{2}}=-\left(s_{0}\right)_{\gamma_{2}}^{2} \log \left(s_{0}\right)_{\gamma_{2}} \mathcal{S}_{\gamma_{2}}, \quad \mathcal{S}_{\gamma_{2}} \sim O(1)
$$

Similarly, when $\gamma_{1}<\gamma_{2}$, for the first branch identifying

$$
q^{2}\left(s_{0}\right)_{\gamma_{1}}^{1-\gamma_{2}} \mapsto w_{11}^{1-\gamma_{2}}
$$

and inserting (5.3a) into the expression for $\tilde{S}_{n u m, \gamma_{2}}$ (C.7),

$$
\left(s_{1}\right)_{\gamma_{2}}=-\left(s_{0}\right)_{\gamma_{2}}^{2} \log \left(s_{0}\right)_{\gamma_{2}} \mathcal{S}_{\gamma_{2}}
$$

For the second,

$$
q^{2}\left(s_{0}\right)_{\gamma_{1}}^{1-\gamma_{1}} \mapsto w_{12}^{1-\gamma_{1}}
$$

and inserting (5.3b) into the expression for $S_{n u m, \gamma_{1}}$ (C.4),

$$
\left(s_{1}\right)_{\gamma_{1}}=-\left(s_{0}\right)_{\gamma_{1}}^{2} \log \left(s_{0}\right)_{\gamma_{1}} \mathcal{S}_{\gamma_{1}} .
$$

Hence, regardless of the relation between the anomaly exponents both branches exhibit proper attenuation magnitude $\left|s_{1} / s_{0}\right|$.

It must be noted that the linear approximation (C.2) is meaningless for divergent roots of the type (5.7), since the first order term $s_{0}$ in (C.2) is the solution of an equal $\gamma$ problem, whose roots always decay.

\section{References}

[1] R. Metzler and J. Klafter, The random walk's guide to anomalous diffusion: a fractional dynamics approach, Phys. Rep., 2000, Vol. 339, No. 1, 1.

[2] G. Drazer and D.H. Zanette, Experimental evidence of power-law trapping-time distributions in porous media, Phys. Rev. E, 1999, Vol. 60, 5858.

[3] T. Kosztołowicz, K. Dworecki, St. Mrówczyński, How to measure sub-diffusion parameters, Phys. Rev. Lett., 2005, Vol. 94, 170602. 
[4] D.S. Banks and C. Fradin, Anomalous diffusion of proteins due to molecular crowding, J. Biophys., 2005, Vol. 89, 2960.

[5] M. Wachsmuth, W. Waldeck and J. Langowski, Anomalous diffusion of fluorescent probles inside living cell nuclei investigated by spatially resolved fluorescence correlation spectroscopy, J. Mol. Biol., 2000, Vol. 298, No. 4, 677.

[6] M. Weiss, H. Hashimoto and T. Nilsson, Anomalous protein diffusion in living cells as seen by fluorescence correlation spectroscopy, J. Biophys., 2003, Vol. 84, 4043.

[7] T.H. Solomon, E.R. Weeks and H. Swinney, Observation of anomalous diffusion and Lévy flights in a two-dimensional rotating flow, Phys. Rev. Lett., 1993, Vol. 71, No. 24, 3975.

[8] B.A. Carreras, V.E. Lynch and G.M. Zaslavsky, Anomalous diffusion and exit time distribution of particle tracers in plasma turbulence model, Phys. Plasmas, 2001, Vol. 8, No. 12, 5096.

[9] P. Manandhar, J. Jang, G.C. Schatz, M.A. Ratner and S. Hong, Anomalous surface diffusion in nanoscale direct deposition processes, Phys. Rev. Lett., 2003, Vol. 90, No. 11, 115505.

[10] J.M. Sancho, A.M. Lacasta, K. Lindenberg, I.M. Sokolov and A.H. Romero, Diffusion on a solid surface: anomalous is normal, Phys. Rev. Lett., 2004, Vol. 92, 250601.

[11] S.B. Yuste, L. Acedo and K. Lindenberg, Reaction front in an $A+B \rightarrow C$ reaction - subdiffusion process, Phys. Rev. E, 2004, Vol. 69, 036126.

[12] T.A.M. Langlands, B.I. Henry and S.L. Wearne, Turing pattern formation with fractional diffusion and fractional reactions, J. Phys. Condens. Matter, 2007, Vol. 19, No. 6, 065115.

[13] Y. Nec and A.A. Nepomnyashchy, Turing instability of anomalous reaction - anomalous diffusion systems, Eur.. J. Appl. Math. ( accepted ).

[14] A. Carpinteri and F. Mainardi, Fractional Calculus in Continuum Mechanics, 1997, SpringerVerlag, New York.

[15] B.I. Henry, S.L. Wearne, Fractional reaction - diffusion, Physica A, 2000, Vol. 276, 448.

[16] B.I. Henry, S.L. Wearne, Existence of turing instabilities in a two-species fractional reaction diffusion system, SIAM J. Appl. Math., 2002, Vol. 62, No. 3, 870.

[17] J.D. Murray, Mathematical Biology, 1989, Springer-Verlag, New York.

[18] B.I. Henry, T.A.M. Langlands, S.L. Wearne, Turing pattern formation in fractional activator - inhibitor systems, Phys. Rev. E, 2005, Vol. 72, 026101.

[19] K.B. Oldham, J. Spanier, The Fractional Calculus, 1974, Academic Press, New York.

[20] Georgiou G., Bahra S.S., Mackie A.R., Wolfe C.A., O’Shea P., Ladha S., Fernandez N. and Cherry R.J., Measurement of the lateral diffusion of human MHC class I molecules on HeLa cells by fluorescence recovery after photobleaching using a phycoerythrin probe, Biophys. J., 2002, Vol. 82, No.4, 1828.

[21] Vigil R.D., Ouyang Q. and Swinney H.L., Turing patterns in a simple gel reactor, Physica A, 1992, Vol. 188, 17. 